\title{
Enhance embodiment of a virtual prosthesis through a training protocol using an EMG-based human-machine interface: a case series
}

\section{Karina Aparecida Rodrigues ( $\sim$ rodrigues.karina@unifesp.br)}

Universidade Federal de São Paulo, Escola Paulista de Medicina, Departamento de Neurologia e Neurocirurgia, Rua Pedro de Toledo, 669, Vila Clementino, Postal Code: 04039-032, São Paulo-São Paulo, Brazil.

\section{João Vitor da Silva Moreira}

Universidade Federal de São Paulo, Escola Paulista de Medicina, Departamento de Neurologia e Neurocirurgia, Rua Pedro de Toledo, 669, Vila Clementino, Postal Code: 04039-032, São Paulo-São Paulo, Brazil.

\section{Daniel José Lins Leal Pinheiro}

Universidade Federal de São Paulo, Escola Paulista de Medicina, Departamento de Neurologia e Neurocirurgia, Rua Pedro de Toledo, 669, Vila Clementino, Postal Code: 04039-032, São Paulo-São Paulo, Brazil.

\section{Rodrigo Lantyer Marques Dantas}

Universidade Federal de São Paulo, Escola Paulista de Medicina, Departamento de Neurologia e Neurocirurgia, Rua Pedro de Toledo, 669, Vila Clementino, Postal Code: 04039-032, São Paulo-São Paulo, Brazil.

\section{Thaís Cardoso Santos}

Universidade Federal de São Paulo, Escola Paulista de Medicina, Departamento de Neurologia e Neurocirurgia, Rua Pedro de Toledo, 669, Vila Clementino, Postal Code: 04039-032, São Paulo-São Paulo, Brazil.

\section{João Luiz Vieira Nepomuceno}

Universidade Federal de São Paulo, Escola Paulista de Medicina, Departamento de Neurologia e Neurocirurgia, Rua Pedro de Toledo, 669, Vila Clementino, Postal Code: 04039-032, São Paulo-São Paulo, Brazil.

\section{Maria Angélica Ratier Jajah Nogueira}

Universidade Federal de São Paulo, Escola Paulista de Medicina, Departamento de Neurologia e Neurocirurgia, Rua Pedro de Toledo, 669, Vila Clementino, Postal Code: 04039-032, São Paulo-São Paulo, Brazil.

\section{Esper Cavalheiro}

Universidade Federal de São Paulo, Escola Paulista de Medicina, Departamento de Neurologia e Neurocirurgia, Rua Pedro de Toledo, 669, Vila Clementino, Postal Code: 04039-032, São Paulo-São Paulo, 
Brazil.

Jean Faber

Universidade Federal de São Paulo, Escola Paulista de Medicina, Departamento de Neurologia e Neurocirurgia, Rua Pedro de Toledo, 669, Vila Clementino, Postal Code: 04039-032, São Paulo-São Paulo, Brazil.

\section{Research Article}

Keywords: human-machine interface, biofeedback, prosthesis, embodiment, proprioception, ownership, agency

Posted Date: July 28th, 2021

DOI: https://doi.org/10.21203/rs.3.rs-757305/v1

License: (c) (i) This work is licensed under a Creative Commons Attribution 4.0 International License. Read Full License 


\title{
Enhanced embodiment of a virtual prosthesis through a training protocol using an EMG-based human-machine interface: a case series
}

\author{
Karina Aparecida Rodrigues, ${ }^{1 *}$, João Vitor da Silva Moreira1, Daniel José Lins Leal Pinheiro', \\ Rodrigo Lantyer Marques Dantas ${ }^{1}$, Thaís Cardoso Santos ${ }^{2}$, João Luiz Vieira Nepomuceno ${ }^{2}$, \\ Maria Angélica Ratier Jajah Nogueira ${ }^{3}$, Esper Cavalheiro', Jean Faber1,2
}

${ }^{1}$ Universidade Federal de São Paulo, Escola Paulista de Medicina, Departamento de Neurologia e Neurocirurgia, Rua Pedro de Toledo, 669, Vila Clementino, Postal Code: 04039-032, São Paulo-São Paulo, Brazil.

${ }^{2}$ Universidade Federal de São Paulo, Instituto de Ciência e Tecnologia, Rua Talim, 330, Vila Nair, Postal Code: 12231-280, São José dos Campos-São Paulo, Brazil.

${ }^{3}$ Centro de Reabilitação Lucy Montoro, Rua Saigiro Nakamura, 600, Vila Industrial, Postal Code: 12220-280. São José dos Campos-São Paulo, Brazil.

* Corresponding author: rodrigues.karina@unifesp.br

\begin{abstract}
Background: The embodiment of a prosthesis can bring a series of benefits during the rehabilitation of people with amputation, such as improvement of motor control and sense of agency, in addition to optimizing the training process with the prosthetic limb. New therapeutic strategies capable of enhancing prosthesis embodiment are, therefore, a key point for better adaptation to and acceptance of prosthesis use. In this study, we developed a system and a new rehabilitation protocol using an EMGbased human-machine interface (HMI) to induce and enhance the embodiment of a virtual prosthesis. Methods: This is a case series with seven people of both sexes with unilateral transfemoral traumatic amputation without previous use of prostheses. Participants performed a training protocol with the EMG-based HMI during the preprosthetic rehabilitation phase, composed of six sessions held twice a week, each lasting thirty minutes. This system was composed of myoelectric control of the movements of a virtual prosthesis immersed in a 3D virtual environment. Additionally, vibrotactile stimuli were provided on the participant's back corresponding to the movements performed. The objectives were to evaluate virtual prosthesis embodiment, to investigate motor learning during training with EMGbased HMI and to determine whether vibrotactile stimuli could facilitate the perception of virtual limb movements. The embodiment was investigated from a set of physiological and behavioral measurements and reports before and after the training. Motor learning was assessed through performance analysis. To investigate the use of vibrotactile stimulation to guide virtual prosthesis movements, the performance was assessed during the virtual prosthesis control test without adding vision.
\end{abstract}


Results and conclusions: The different features evaluated throughout the protocol training consistently showed the induction and enhancement of virtual prosthesis embodiment and increased motor control. Therefore, this protocol using EMG-based HMI was shown to be a viable option to achieve the embodiment of a virtual prosthetic limb and to train motor control. Furthermore, the participants were able to guide the prosthesis based on vibrotactile stimuli, showing that this method can be used as an alternative sensorial path to be implemented in new therapeutic strategies and neuroprostheses to facilitate the movement perception of a prosthetic limb.

Keywords: human-machine interface, biofeedback, prosthesis, embodiment, proprioception, ownership, agency

\section{Introduction}

The incidence of lower limb amputations around the world varies between 2.8 and 43.9 per 100,000 population/year [1]. The indication for prosthesis use after an amputation procedure aims to recover functionality and autonomy, providing a better quality of life [2]. However, even with multidisciplinary interventions and technological advances in the development of prostheses, a considerable portion of patients do not fully adapt to the use of a prosthetic limb [3-6]. Different factors may contribute to this nonadaptation associated with prosthesis use, including aesthetic, ergonomic, functional, psychological, and cognitive aspects [5-7].

In this way, searching for therapeutic strategies and approaches that help the adaptation and acceptance process of prosthetic limb use is particularly important. Recent research has pointed out that a key point during this process is prosthesis embodiment, which can bring a series of benefits, such as facilitation of the learning process and motor control, adaptation to and acceptance of prosthetic limb use [8-9]. The concept of embodiment of an external object can be defined as "the ability to process properties of this object at the sensory, motor and/or affective levels in the same way that the properties of one's own body parts" [9-10]. Several studies have corroborated this concept, showing that people with amputation can have a better perception of the prosthesis when it is voluntarily controlled and/or provides somatosensory feedback [8, 11-13].

Based on the premises of voluntary control and somatosensory feedback, EMG-based humanmachine interface (HMI) training provides a real-time paradigm for the study of the embodiment of an assistive device. Additionally, it can be used as a complementary therapeutic option to conventional treatments [14]. In this study, a new training protocol was developed using an EMG-based HMI applied during the preprosthetic rehabilitation phase of people with transfemoral amputation. The EMG-based HMI was designed in a way that the participants could control the movements of a prosthesis immersed 
in a virtual reality (VR) environment using the myoelectric activity of the stump while receiving noninvasive vibrotactile stimuli applied on their back, which were mapped to represent the movements of the virtual prosthesis.

This system and protocol training aggregated previous knowledge of the literature, but they have not still been applied and integrated in the clinical context during the rehabilitation of people with amputation (myoelectric control [15-16], VR environment [17-18] and vibrotactile stimulation [1921]).

Prosthetic myoelectric control has been widely explored in research and clinical environments $[15-16,22]$. Some studies have also used the myoelectric control of virtual prostheses mainly in the pretraining phase before the use of physical prostheses [17, 23]. However, in general, these studies do not use an immersive virtual environment and focus on control conditions and not necessarily on the closed loop between control and feedback, as we propose in this work.

Training protocols using visual feedback in immersive VR environments have shown promising effects in a variety of clinical contexts [17-18, 24-25] and in the induction of the embodiment of a body, limb or virtual object [26-29]. Because the learning acquired in a virtual environment is transferable to the physical environment [17-18, 25], it can also optimize adaptation to the use of a physical prosthesis.

Although vibrotactile stimulation is often used as a way of providing feedback on prosthetic limbs, in general, it is used to represent tactile information [19-21]; here, we propose vibrotactile stimulation to represent movement [29]. One of the great challenges in the rehabilitation of people with amputation is how to provide lost sensory information again [11]. Most current lower limb prostheses do not provide sensory feedback, which makes the user largely dependent on vision to determine the prosthetic limb position and its interaction with the environment. Furthermore, reestablishing proprioceptive sensory information related to movement perception is crucial for the development of embodiment [30-31] and improvement of motor control [30, 32].

The hypothesis was that training with this EMG-based HMI, combining voluntary control with immersive visual and vibrotactile feedback, could induce virtual prosthesis embodiment and train the stump muscles involved in virtual limb control. In addition, we expected that vibrotactile stimuli would facilitate the perception of virtual prosthesis movements without the aid of vision.

To evaluate these hypotheses, we analyzed a series of physiological and behavioral responses and reports associated with the embodiment process of a virtual prosthesis throughout the training protocol. In addition, we assessed the individual's performance during the execution of motor tasks along the training with the EMG-based HMI. Finally, we systematically explored whether vibrotactile stimulation can be used as an alternative sensorial path to map the movements of the virtual prosthesis. 


\section{Materials and Methods}

\section{Experimental design}

This is a case series study that assessed a) as a primary outcome, the induction of embodiment of a virtual prosthesis through a training protocol with an EMG-based HMI and b) as secondary outcomes, the ability to control the virtual prosthesis during training with EMG-based HMI and the use of vibrotactile stimuli in the perception of virtual limb movements. The research protocol was approved by the Ethics and Research Committee of the Universidade Federal de São Paulo $\left(\mathrm{n}^{\circ}\right.$ 3.030.942) and of the Hospital Municipal Dr. José de Carvalho Florence ( $n^{\circ}$ 3.273.170), Brazil.

\section{Participants}

For the inclusion of participants in the research, the following criteria were adopted: people with unilateral transfemoral amputation, both sexes, age between 18 and 59 years, and without previous use of prostheses. People who had open skin lesions on the stump or back, deficit uncorrected visual impairment and associated neurological diseases were excluded from participation in the study. Seven people participated in the study ( 6 men and 1 woman; mean age \pm standard deviation (SD): 34.7 \pm 9.9 years; height: $1.75 \pm 0.07 \mathrm{~m}$; body mass: $69.3 \pm 14.4 \mathrm{~kg}$; amputation time: $19.7 \pm 24.6$ months).

The demographic, physical, cognitive, and psychological assessments of all participants are listed in Table 1 and Additional file 1. The participants provided written consent prior to the start of the study, and all ethical recommendations and regulations were followed. 
Table 1 - Characterization of the demographic, physical, cognitive, and psychological aspects of the participants.

\begin{tabular}{|c|c|c|c|c|c|c|c|}
\hline \multirow[t]{2}{*}{ Measures } & \multicolumn{7}{|c|}{ Participants } \\
\hline & A & B & C & D & E & $\mathrm{F}$ & G \\
\hline Age (years) & 46 & 32 & 22 & 32 & 24 & 46 & 41 \\
\hline Sex & Male & Male & Male & Male & Male & Female & Male \\
\hline Education (years) & 11 & 11 & 11 & 7 & 5 & 11 & 5 \\
\hline Height $(\mathrm{m})$ & 1.69 & 1.87 & 1.81 & 1.79 & 1.69 & 1.67 & 1.75 \\
\hline Body mass (kg) & 80.9 & 64.5 & 60.0 & 88.3 & 46.0 & 78.7 & 67.0 \\
\hline Amputation time (months) & 11 & 3 & 11 & 74 & 21 & 13 & 5 \\
\hline Amputation side & Right & Right & Left & Right & Left & Right & Right \\
\hline Stump length $(\mathrm{cm})^{* 1}$ & 34 & 35 & 37 & 13 & 20 & 37 & 30 \\
\hline Stump pain intensity (numerical pain scale)*2 & 8 & 3 & 0 & 0 & 0 & 0 & 0 \\
\hline Phantom limb sensation & No & Yes & Yes & No & Yes & Yes & No \\
\hline $\begin{array}{l}\text { Stump hip muscle strength }(\mathrm{Kg} / \mathrm{F})^{* 3} \\
\text { Flexors }\end{array}$ & 29.2 & 17.5 & 14.2 & 16.6 & 14.7 & 15.7 & 17.0 \\
\hline Extenders & 20.5 & 9.6 & 13.9 & 12.8 & 12.9 & 14.1 & 12.8 \\
\hline Abductors & 14.8 & 11.4 & 12.2 & 17.2 & 11.3 & 13.7 & 12.4 \\
\hline Adductors & 15.0 & 10.1 & 12.2 & $* *$ & 9.9 & 11.3 & 10.8 \\
\hline Functional level (AMPnoPRO)*4 & 34 & 34 & 37 & 37 & 39 & 27 & 30 \\
\hline Physical activity level (IPAQ) ${ }^{* 5}$ & High level & Low level & High level & High level & High level & High level & High level \\
\hline Cognitive level (MoCA)*6 & 26 & 28 & 23 & 23 & 20 & 29 & 18 \\
\hline Depression level (HAD)*7 & 5 & 2 & 4 & 8 & 1 & 7 & 1 \\
\hline Anxiety level (HAD)*7 & 4 & 5 & 7 & 6 & 2 & 1 & 3 \\
\hline
\end{tabular}

*1 Stump measurement reference was made considering the distance from the greater trochanter of the femur to the distal extremity [33]

*2 Numerical pain scale, where '0' means no pain and '10' means the worst pain [34-35].

*3 Measurement made using a digital dynamometer. The point of force application was considered the midpoint of the stump length. Three isometric contractions were performed for each muscle group, and the mean peak strength was calculated over the last $5 \mathrm{~s}$ of contraction [36].

*4 The Amputee Mobility Predictor No Prosthesis (AMPnoPRO) assesses mobility aspects of amputees and predicts functional levels related to the use of prostheses [37].

*5 The International Physical Activity Questionnaire - short version (IPAQ) was used to assess the level of physical activity [38].

*6 The Montreal Cognitive Assessment (MoCA) was used to assess cognitive functions [39].

*7 The Hospital Anxiety and Depression (HAD) Scale was used to assess levels of anxiety and depression [40].

** For participant 'D', it was not possible to assess the strength of the adductor muscles due to the small size of the stump.

\section{EMG-based human-machine interface}

The EMG-based HMI was designed to work using the electrophysiological activity of the muscle on the stump. Through a real-time recording and processing of this activity, the participants were able to control the knee movements of a virtual prosthesis while receiving patterns of vibrotactile stimulation on their back, representing the current position of the virtual prosthesis (Figure 1). 
Recording of muscle activity. The activity of the hip flexor and extensor muscles on the stump was recorded using surface electromyography (EMG) (Figure 1-A.1). Due to the surgical procedure and muscle reinsertion, we were unable to say precisely which muscle was being recorded. Therefore, we established the criteria of using a hip flexor and a hip extensor muscle; once under normal conditions, these muscles are also recruited during knee extension and knee flexion, respectively [41]. Electrode placement for each muscle and participant was determined by applying excitomotor electrical current stimulation and visualizing the muscle contraction response. These positions were mapped for each person and used in all training sessions. Two channels of an Intan Technologies® chip were used to amplify the electrophysiological signals, and the chip was connected to the OpenEphys ${ }^{\circledR}$ analog-digital converter board in communication with its software [42-43]. The electrophysiological signals were sampled at a rate of $10 \mathrm{kHz}$.

Real-time processing. Data were processed using MATLAB $^{\circledR}$ (R2017b). For real-time control, every $200 \mathrm{~ms}$, the EMG signals in each channel were loaded in blocks of 5120 samples, resulting in a $60 \%$ overlap with the previous sample [44]. Then, the samples were filtered using a twentieth-order IIR bandpass filter in the frequency range from 10 to $500 \mathrm{~Hz}$ and filtered at $60( \pm 2)$ $\mathrm{Hz}$ with its harmonics [45]. Then, the EMG signal in each window was resampled to $2 \mathrm{kHz}$, and its root mean square (RMS) was calculated to estimate the muscle contraction level [46].

To control feedback, two criteria needed to be satisfied: a) Agonist muscle activation threshold. The RMS of the agonist muscle signals had to be greater than $2 \mathrm{SD}$ in relation to the baseline signal for the system to recognize the direction of movement (knee extension or flexion). b) Relationship of activation between agonist and antagonist muscles. Initially, the RMS of the antagonist muscle could not exceed $80 \%$ in relation to the agonist (this variable was also used as a criterion for the progression in difficulty levels during training). Therefore, a higher level of activity associated with the hip flexor muscle resulted in knee extension movement of the virtual prosthesis and, simultaneously, in an upward vibrotactile stimulation pattern. A higher level of activity associated with the hip extensor muscle resulted in knee flexion of the virtual prosthesis and, simultaneously, in a downward vibrotactile stimulation pattern (Figure 1-A.2). For more details about the definition of the vibrotactile stimulation pattern, see Additional file 2. Another set of control criteria was adopted in relation to the movement speed of the virtual prosthesis, and the criteria were determined by the estimated contraction level of the agonist muscle: RMS values that exceeded 2 SD in relation to the baseline signal were considered the minimum activity, and RMS values of the maximum voluntary isometric contraction (MVIC) used to normalize the signals of each muscle [46] were considered the maximum activity. Therefore, the greater the contraction force exerted by the agonist muscle, the faster the movement of the virtual prosthesis. 
Virtual reality environment. The virtual environment was developed on the Unity3D® platform (2018.4). The environment was conceived to simulate a regular clinical room where the users would see themselves as a humanoid avatar using a transfemoral prosthesis in the corresponding lower limb. The subjects were able to control the knee extension and flexion movements of the prosthetic limb within a range between $0^{\circ}$ and $90^{\circ}$ (Figure 1-B.1). Moreover, the virtual environment was designed to enable gamification of the protocol with different stages and motivational messages to reinforce learning. The participants accessed the virtual environment using a Samsung® Odyssey Oculus Head-Mounted Display (HMD) that provided a first-person view in a fixed sitting position [26$27]$ and the ability to visually explore the whole $3 \mathrm{D}$ virtual environment.

Vibrotactile stimulation device. A total of 16 vibrotactile actuators (10 mm x $6 \mathrm{~mm}$; $5 \mathrm{~V}$-DC) were assembled in a 4x4 matrix and positioned on the subject's back [47], with an average distance of $6 \mathrm{~cm}$ among them. Vibrotactile stimulation was applied at frequencies between 260 and $330 \mathrm{~Hz}$, which is optimal for stimulating Pacinian corpuscles, the main skin vibration receptors [48]. Vibrotactile actuators were arranged in groups of 4 (organized by rows on the back), and each group was activated together (actuator activation was performed through an Arduino® platform communicating in real time with MATLAB® (R2017b) and carried out by a transistorized circuit, 2n2222 NPN, through a pulse width modulation (PWM) signal). All mini-motors placed along the same row vibrated with the same intensity, with maximum intensity when the virtual prosthesis was positioned at a specific movement angle $\left(0^{\circ}, 30^{\circ}, 60^{\circ}\right.$, or $\left.90^{\circ}\right)$; there was a vibratory overlap of $30^{\circ}$ with the adjacent rows to produce a continuity effect on vibratory perception [44] (Figure 1-B.2). 
A

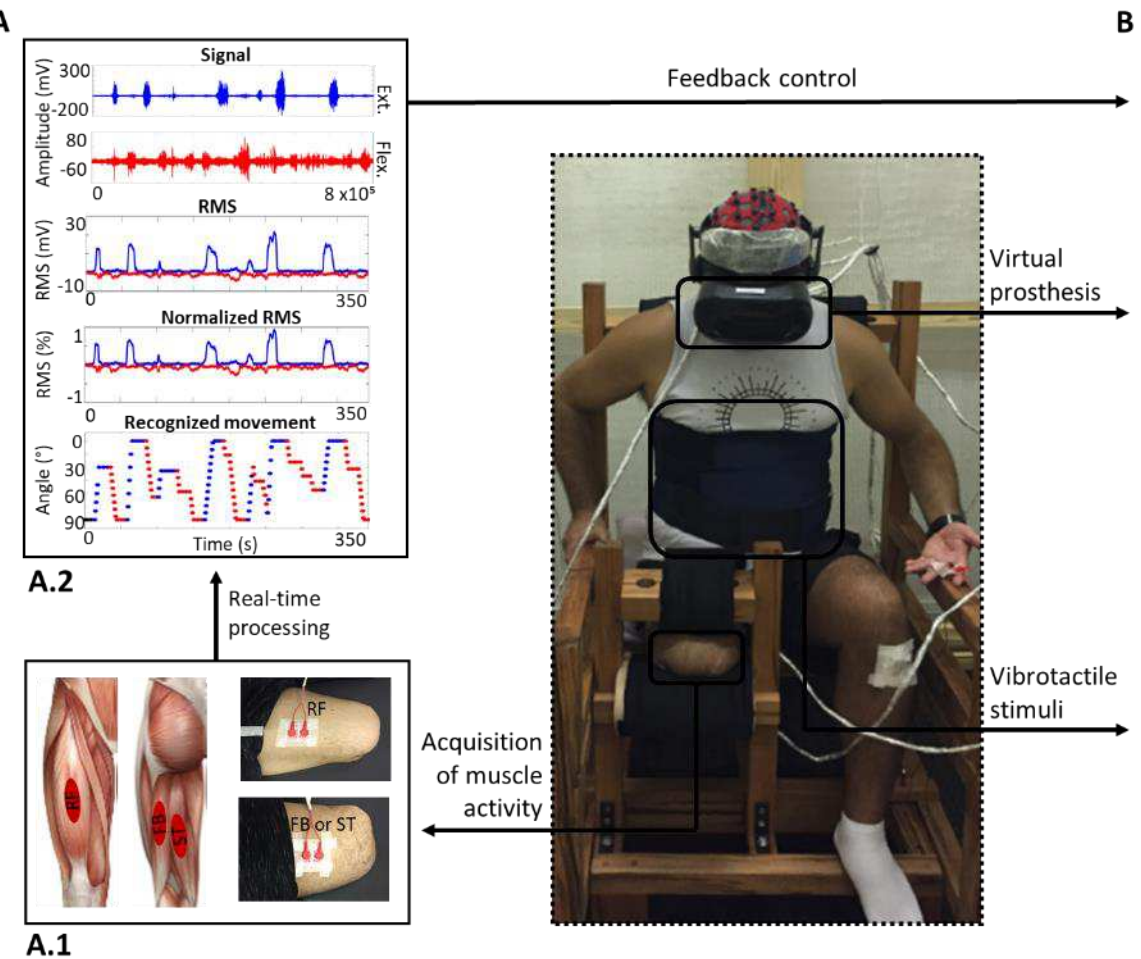

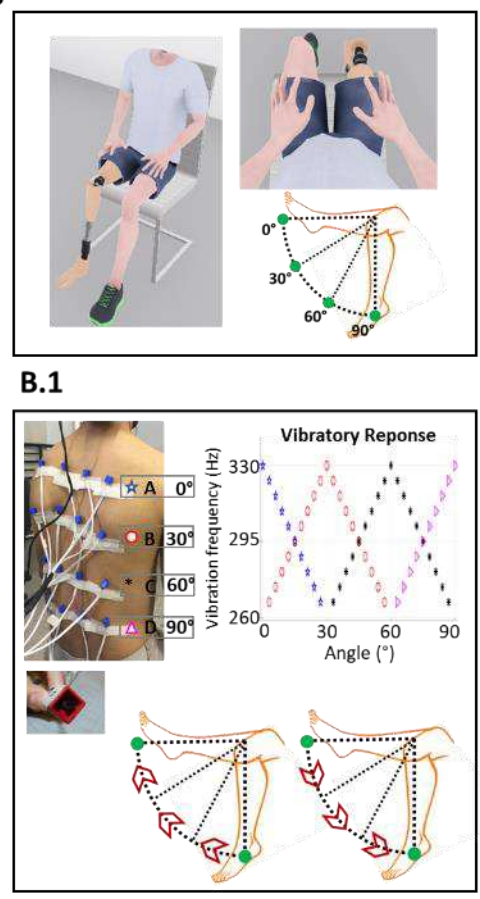

B.2

Figure 1 - EMG-based human-machine interface scheme. A) Muscle activity recording through a surface EMG. A.1) Illustration of the rectus femoris (RF) (hip flexor and knee extensor), femoral biceps long head (FB) and semitendinosus (ST) muscles (hip extensors and knee flexors) and positions of the surface electrodes on these muscles responsible for controlling the movements of the virtual prosthesis knee. A.2) Schematic diagram of the real-time processing of electromyographic activity and RMS calculations to estimate the level of muscle contraction. The RMS was normalized by the MVIC of each muscle. Regarding recognition of the movement direction, the activity of the agonist muscle should be twice as high as the average of the baseline signal, and the antagonist muscle could not exceed a threshold relative to the agonist, which was initially set at $80 \%$. The recognized EMG patterns were mapped into visual and vibrotactile feedback. B) Feedback. B.1) Visual feedback. Avatar modeled with a transfemoral prosthesis and visualization from the first-person perspective are shown. The range of motion available to the prosthetic knee was set between $0^{\circ}$ and $90^{\circ}$. B.2) Vibrotactile feedback scheme. The positioning of vibrotactile actuators on the participant's back was organized in a $4 \times 4$ matrix. The paradigm for the applied vibratory stimuli was associated with the movements of the virtual prosthesis: upward vibration during knee extension and downward vibration during knee flexion. The vibratory intensity peak of a given row corresponded to a specific angle of knee movement (row $\mathrm{A}, 0^{\circ} ; \mathrm{B}, 30^{\circ} ; \mathrm{C}, 60^{\circ}$; and $\mathrm{D}, 90^{\circ}$ ), with an overlap of $30^{\circ}$ between adjacent rows.

\section{Training protocol}

Each session consisted of the following steps: a) the bipolar surface electrodes $(\mathrm{Ag} / \mathrm{AgCl})$ were positioned for EMG recording on the stump hip flexor and extensor muscles (the reference electrode was positioned on the tibial tuberosity of the opposite limb) after skin preparation with local trichotomy and asepsis with $70 \%$ alcohol to decrease the local impedance [49]; b) the participants were 
positioned in the chair, where they remained throughout the whole training protocol; c) the electrodes were placed for electroencephalographic (EEG) recording (the reference electrode was positioned on the mastoid process on the right side) [50-51]; d) the vibrotactile actuators were positioned on the subject's back; e) hearing protectors were used to minimize noise interferences from the external environment, including the noise produced by the vibrotactile actuators; and f) the HMI was calibrated by recording the EMG basal activity and MVIC relative to the muscles involved in controlling the knee movements of the virtual prosthesis (Figure 2-A).

Two preliminary sessions were conducted prior to the start of the training protocol to familiarize participants with the EMG-based HMI. In these sessions, the participants learned to associate the stump muscular contraction with virtual prosthesis movements (for details, see Additional file 3). After this stage, the training was based on an operant conditioning paradigm, in which there was a progressive increase in the difficulty of the tasks with contingent feedback and rewards to reinforce learning. Overall, contingent feedback itself has a positively reinforcing effect, but this was supplemented with motivational messages, such as "congratulations", at the end of each task block $[48,52]$.

In total, six training sessions lasting 30 minutes each and consisting of tasks involving motor control were conducted twice a week. For each task, the participants moved the virtual prosthesis until they reached a specific predetermined position set at four target angles: $0^{\circ}, 30^{\circ}, 60^{\circ}$ or $90^{\circ}$ (a combination of angles with targets at $0^{\circ}, 45^{\circ}$ and $90^{\circ}$ was also used as a preliminary stage for each new level of difficulty). To guide the movements in real time, the participants were presented with a visual clue (semicircular ruler) indicating the position to which they should move the virtual prosthesis (Figure 2-B).

The following criteria were adopted to increase the task difficulty: a) Tolerance of contraction of the antagonist muscle. Antagonist muscle activation up to $80 \%$ in relation to agonist was initially established, which decreased by $10 \%$ at each new difficulty level; b) Precision of movement. For a task to be considered correctly performed, a range of positions was adopted in relation to the target angle. The difficulty levels varied from the target angle as follows: $\pm 15^{\circ}, \pm 10^{\circ}$ and $\pm 5^{\circ}$. Therefore, initially, there was no need for refined muscle control (regarding the isolation of agonist muscle contraction) and movement precision; however, this became necessary as the difficulty gradually increased (Figure 2-C).

In this manner, given a particular difficulty combination (tolerance of contraction of the antagonist muscle and precision of movement), the participants performed a preliminary block and then a task block composed of a set of target angles, $0^{\circ}, 30^{\circ}, 60^{\circ}$ and $90^{\circ}$ (each presented randomly 
four times), for a total of sixteen tasks for each block. After an attempt of $20 \mathrm{~s}$ or if the target angle was hit, the next task was presented (if the participant did not hit the task within $20 \mathrm{~s}$, it was considered a failure, although the participant was not informed about the failure). The performance was assessed at the end of the task block, and the difficulty was increased if the participant had a success rate of $75 \%$ or more; otherwise, the same difficulty combination was performed again.

A

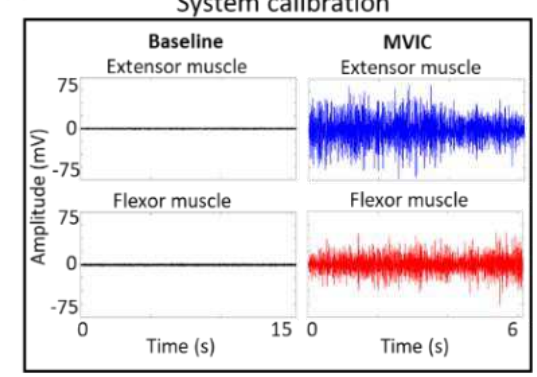

A.1

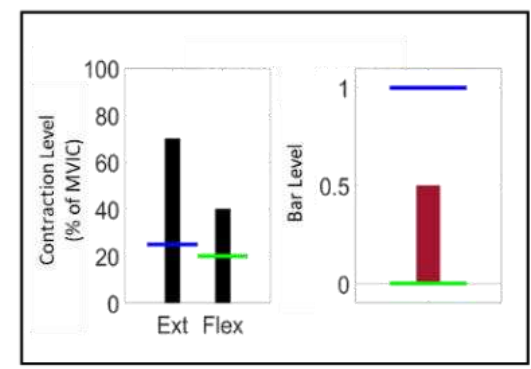

A.2
B

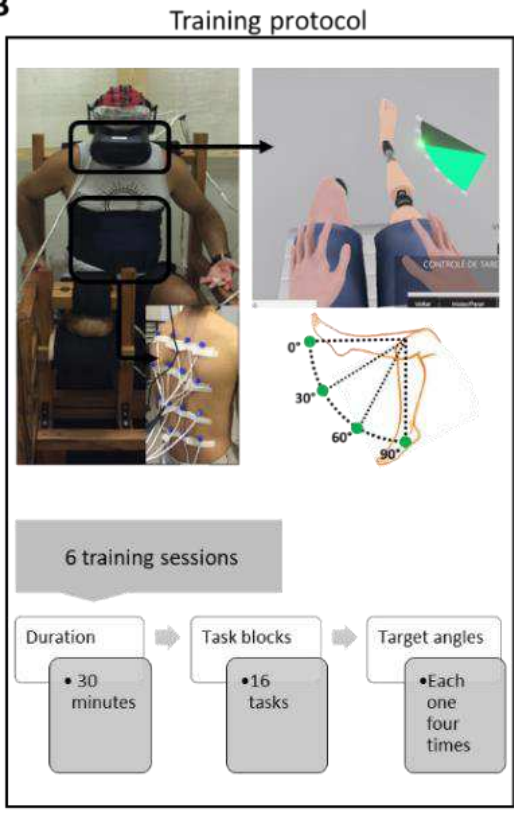

C

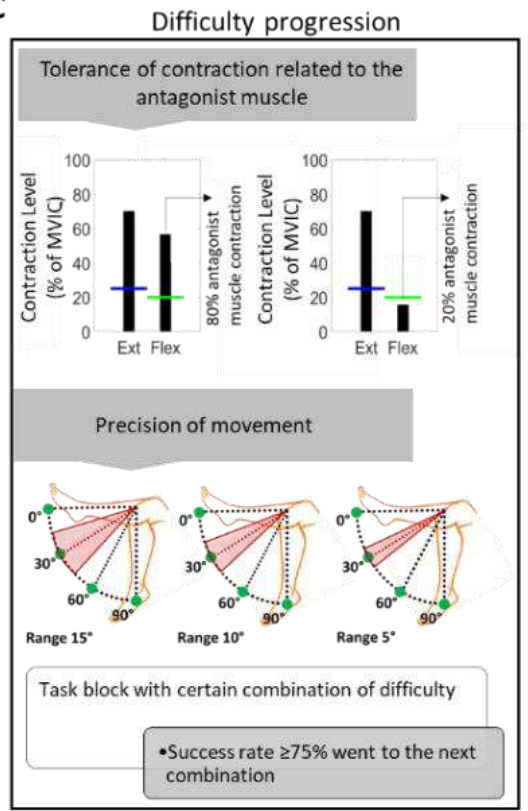

Figure 2 - Training protocol with EMG-based HMI scheme. A) System calibration. A.1) Acquisition of basal muscle activity ( $15 \mathrm{~s}$ with relaxed muscles). To record the MVIC, three repetitions were performed for each muscle, with $6 \mathrm{~s}$ and $45 \mathrm{~s}$ of rest between each repetition. A.2) Test to verify the proper functioning of the system with visual feedback on the screen. Visualization of the contraction level relative to each muscle performing knee extension and knee flexion of the virtual prosthesis (represented by the red bar: rising bar $\rightarrow$ knee extension; descending bar $\rightarrow$ knee flexion). The blue and green horizontal bars are thresholds for the system to recognize the signal as a voluntary contraction. B) Training protocol diagram. Feedback within the virtual environment consisted of visual clues indicating the target angles that the participants had to reproduce. The target angles used were $0^{\circ}, 30^{\circ}, 60^{\circ}$ and $90^{\circ}$. Each angle was randomly presented four times during each task block (the participant had $20 \mathrm{~s}$ to establish each target angle). In addition to visual feedback, the participants received concomitant vibrotactile feedback on their back. The training sessions lasted thirty minutes, and within that time, as many task blocks as possible were performed. C) Difficulty progression. Two criteria were adopted to increase the difficulty: i) Tolerance of contraction related to the antagonist muscle. Initially, the antagonist muscle could have up to $80 \%$ activation in comparison to the agonist muscle. The tolerance decreased progressively by $10 \%$ at each new difficulty level (the lower the tolerance was, the greater the need to isolate the agonist muscle contraction). ii) Precision of movement. To evaluate if a target angle has been reached, different ranges of prosthesis position, in relation to the target angle, were adopted $\left(15^{\circ}, 10^{\circ}\right.$, and $5^{\circ}$ : the lower the range was, the greater the 
necessary precision of movement). Given a tolerance of contraction related to the antagonist muscle, the different precision difficulties were progressively combined. If the participant had a success rate $\geq 75 \%$ on a task block with a certain combination of difficulties, the next block instituted a new combination of difficulties.

\section{Embodiment and behavioral assessment}

We assessed a set of measurements at the beginning and end of the experimental protocol to examine the induction of virtual prosthesis embodiment (Figure 3). This test set was selected based on affective, spatial perception, and motor mechanisms. These three features were proposed by De Vignemont (2011) and underlie the development of the object's embodiment:

a) Affective measurement - skin conductance response (SCR) was used to detect inherent physiological responses when the virtual prosthesis was threatened [53-55].

b) Spatial perception measurement - a crossmodal congruency task (CCT) was used to identify visuotactile interference in the peripersonal space [28-29,56].

c) Motor measurement - the participants' performances during the training were used to assess their ability to control the virtual prosthesis [10]. This analysis also contemplates the secondary objective of investigating the ability of motor control throughout the training related to the learning process [57].

d) Quantification of the ownership and agency sense with regard to the virtual prosthesis through a numerical scale to assess body self-perception [54].

e) Recording of resting-state EEG activity to evaluate eventual functional changes expressed through electrophysiological brain patterns [58]. 


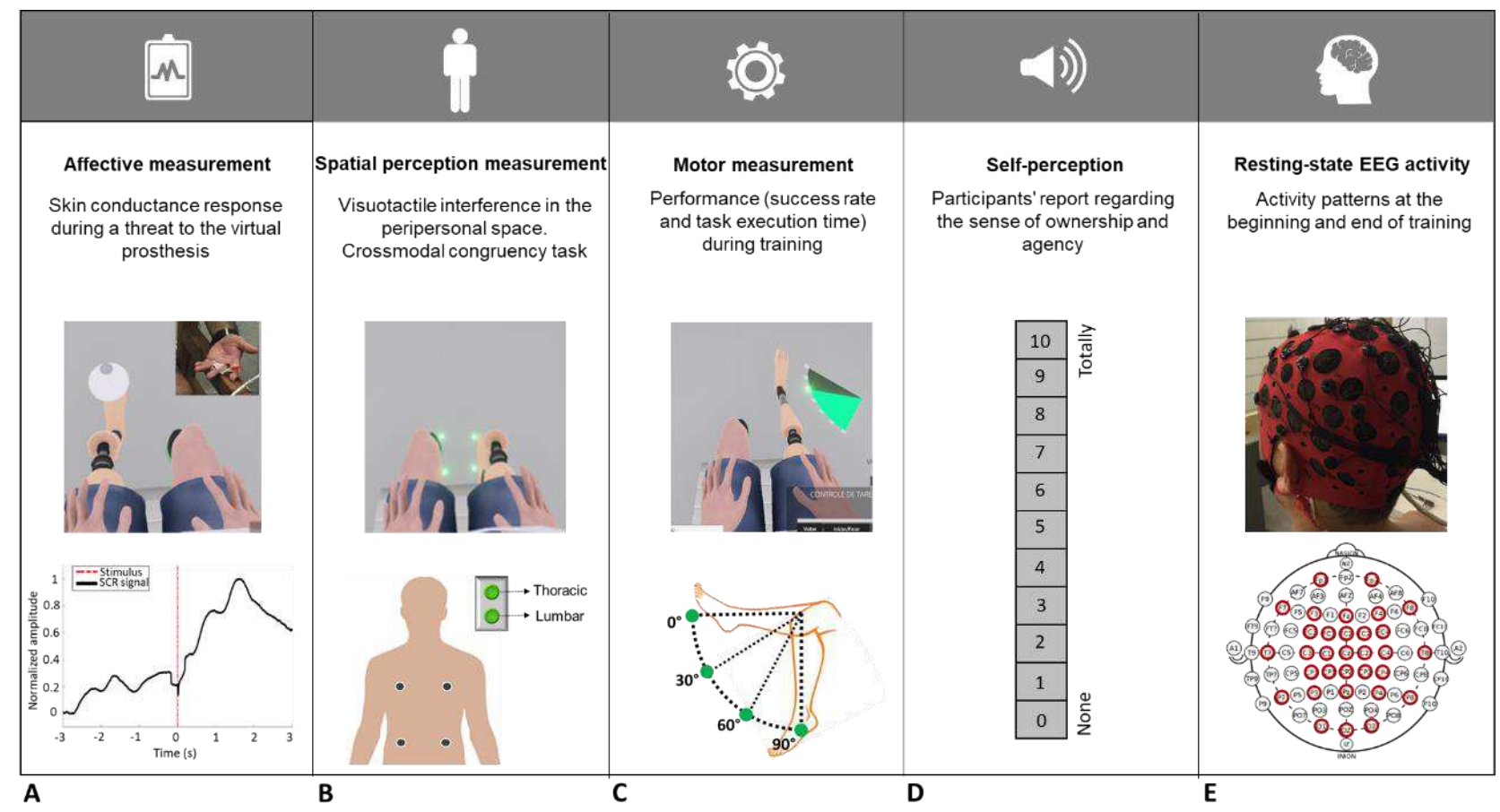

Figure 3 - Embodiment and behavioral assessment. A) Affective measurement. For the measurement of the skin conductance response, two surface electrodes were placed on the intermediate phalanges of the second and third left hand fingers, and the SCR was recorded once a chandelier dropped on the virtual prosthesis, representing a threatening stimulus. B) Spatial perception measurement. Crossmodal congruency task. The protocol consisted of visualizing the prosthesis movements with (VR + VT) or without (VR only) concomitant vibrotactile stimulation and subsequent performance of the CCT. During the CCT, visual stimuli were applied within the VR environment close to the avatar's feet (close to the hallux or heel) soon after the appearance of the visual distractor, and a vibratory stimulus was applied on the participant's back (thoracic or lumbar). The CCT was composed of sixteen different combinations of visual and vibrotactile stimuli, each presented four times at random, for a total of sixty-four trials. The participants were instructed to press a button corresponding to the location on their back where they received the vibratory stimulation as quickly as possible while ignoring the visual distractor. C) Performance (success rate and execution time) during the different levels of difficulty of the training with EMG-based human-machine interface. D) Self-perception. Quantification of the ownership and agency sense with regard to the virtual prosthesis through a numerical scale. E) Resting- state EEG activity. Thirty-two surface electrodes were placed on participants' scalps to record resting-state activity for two minutes with their eyes open at the beginning of each session.

Furthermore, at the end of the training protocol, we applied a test based on the same protocol as the training but without visual stimuli to assess the perception of virtual prosthesis movements from vibrotactile stimuli. During all the assessments described above, the participants were arrayed as shown in Figure 1. 
Affective measurement. SCR is a physiological measure that allows indirect assessment of the activity of the sympathetic autonomic nervous system (SNAS) related to changes in emotional state during specific events $[53,59]$. SCR acquisition was accomplished using the e-Health ${ }^{\circledR}(2.0)$ system coupled to an Arduino Uno®, with a sampling rate of $20 \mathrm{~Hz}$. The SCR recording was performed at the initial session and at the penultimate training session; for this, surface electrodes $(\mathrm{Ag} / \mathrm{AgCl})$ were placed on the intermediate phalanx of the second and third left hand fingers [19]. This recording was made 2 minutes before and during the simulation of a threat - a chandelier falling on the virtual prosthesis [60] (Figure 3-A). At the beginning of the training sessions, all participants watched a video showing the fall of the chandelier on the virtual prosthesis, and they were informed that at some point during the sessions, the same event could occur, thereby minimizing the effects of surprise on the measurements [55]. The participants did not know on which day this test would be conducted. Finally, the magnitude of the SCR was analyzed [59].

Spatial perception measurement. The evaluation of the extension of peripersonal space by including the virtual prosthesis was based on the analysis of the crossmodal congruency effect (CCE) during a CCT: visual stimulus (given by visual distractors onto the virtual body) and a concomitant tactile stimulus (given by the vibratory stimuli onto the participant's back) [28-29]. To perform this task, the participants visualized the lower limbs of the avatar and a luminous point (visual distractor) in four different positions: on either side of the hallux or heel. In addition, four vibrotactile actuators were positioned on the participants' backs: two were placed in the thoracic region, and two were placed in the lumbar region on both sides. Therefore, there were 16 possible stimulus combinations: 4 positions of the visual distractors and 4 positions related to the vibratory stimuli, and each combination was randomly presented four times for a total of 64 repetitions in each task block. A visual distractor was presented and followed $100 \mathrm{~ms}$ later by vibrating stimulation for $350 \mathrm{~ms}$. The participants were then instructed to press a button based on the place on their back that they had received the vibratory stimulation while ignoring the visual distractor. They had two options: upper (thoracic) or lower (lumbar). If the participant did not press the button within $2 \mathrm{~s}$, the next combination was presented [29] (Figure 3-B).

In summary, the CCT protocol consisted of observing the virtual prosthesis performing knee flexion and extension movement (at an angular speed of $45 \%$ for 1 minute) with or without concomitant vibratory stimulation related to virtual prosthesis movements. This observation sequence was random, and the CCT task block was performed after each paradigm. All participants previously underwent training and started this task only after reaching an accuracy of $85 \%$ in localizing the vibratory stimulus [29]. In this manner, the CCE was calculated as the difference in the reaction time between incongruent (for instance, when a visual distractor was localized on the upper part of the foot 
and the vibratory stimulation was in the lumbar region) and congruent conditions (for instance, when a visual distractor was localized on the upper part of the foot and the vibratory stimulation was in the thoracic region) [29, 61].

Motor measurement. Analysis of the execution time and success rate in tasks during training with the EMG-based HMI system was performed while considering the different levels of difficulty during the tasks, the tolerance for antagonist muscle contraction and the precision of movement.

Self-perception. The sense of ownership can be defined as our ability to perceive our own body and to differentiate it from other bodies or objects using sensory information [62]. The sense of agency, in contrast, is related to the perception of control of one's own body movements and distinguishing our actions from those of other people or objects [63]. The participants quantified on a scale from 0 to 10 , where 0 indicated "nothing" and 10 indicated "totally", how much they felt the virtual prosthesis was part of their own body and how much they felt that they could control it [54].

Resting-state EEG activity. EEG activity was noninvasively recorded during all training sessions. An Intan Technologies ${ }^{\circledR}$ chip was used to amplify the electrophysiological recordings and was connected to an OpenEphys ${ }^{\circledR}$ analog-digital converter board with communication with OpenEphys ${ }^{\circledR}$ software [42-43]. The sampling rate was set at $10 \mathrm{kHz}$. Thirty-two electrodes were positioned on the participants' scalps following the recommendations of the International System 1010 [51]. Resting-state activity was recorded from the participants for 2 minutes with their eyes open at the beginning of each training session (Figure 3-D). For subsequent analyses, the recorded signals in the first and last training sessions were considered.

Perception of virtual prosthesis movements using vibrotactile stimuli. A test with a similar arrangement to the EMG-based HMI training sessions was performed. In this test, the participants were asked to accomplish the same tasks by voluntarily controlling the virtual prosthesis and achieving the target angles but now using vibratory stimulation patterns as the guide without the aid of vision. For each task, a visual clue associated with the target angle was presented to the participant; subsequently, the screen of the Oculus HDM was turned off, and the participant had to use the vibratory stimuli on the back to guide their movements and reach the target angle. The target angles were defined as $0^{\circ}, 30^{\circ}, 60^{\circ}$ and $90^{\circ}$, and each angle was presented four times at random during each task block, for a total of 16 tasks per block. The same criteria for movement precision were adopted $\left(15^{\circ}, 10^{\circ}\right.$ and $5^{\circ}$ variation in position relative to the target angle). Four task blocks were performed for each of the 3 levels of difficulty associated with the precision of movements (Figure 3-C). Finally, variables related to performance during this test, execution time and success rate were analyzed. 


\section{Electrophysiological signal processing}

Regarding the SCR signal analysis, the following steps were performed: a) smoothing the original $x(t)$ signal by averaging it over a 3-s sliding window with $50 \%$ overlap along the whole signal and producing an $x^{\prime}(\mathrm{t})$ signal; b) calculating the phase signal from the difference $y(t)=x(t)-x^{\prime}(t)$; and c) applying a logarithmic scale over the magnitude of the signals and considering the $3 \mathrm{~s}$ of signal before and $3 \mathrm{~s}$ after the application of the visual stimulus (i.e., the moment when the chandelier enters the visual field of the participant within the VR environment) [59]. The SCR signals from participant "B" were excluded from the analysis due to noise issues during registration.

We developed a MATLAB ${ }^{(R 2017 b)}$ routine to process the EEG signals, which interfaced with the EEGLab toolbox. First, signal preprocessing was performed, which consisted of a) signal resampling from $10 \mathrm{kHz}$ to $1 \mathrm{kHz}$; b) filtering with an FIR bandpass filter that considered only the range between $4 \mathrm{~Hz}$ and $45 \mathrm{~Hz}$ [64]; and c) visually inspecting the signals from each channel over time. All temporal periods with evident noise (i.e., body and eye movement artifacts) were excluded. In this step, the FC4 channel was removed from the study due to the presence of noise with high amplitude throughout the measurements; and d) common average reference (CAR) spatial filtering was applied to remove other common noise between channels. The filtered signals were evaluated in the frequency domain; for this, we calculated the power spectral density (PSD) using an adapted version of Welch's technique with $0.5 \mathrm{~s}$ windows and $50 \%$ overlap [65].

\section{Statistical analysis}

The analyses of the data and electrophysiological signals were performed in MATLAB® (R2017b) using its statistical toolbox and algorithms. Initially, the Kolmogorov-Smirnov (KS) normality test was applied to evaluate the distribution of the data. Parametric or nonparametric hypothesis tests were used based on the classification of the KS test [66]. Differences were considered significant when $p<\alpha$, where $\alpha=0.05$.

To compare SCR magnitudes among the 4 different periods (pre- and postthreat in the initial and final sessions), a two-way ANOVA was used with a Tukey-Kramer post hoc correction. A oneway MANOVA was applied followed by canonical discriminant analysis to determine whether the set of variables (SCR amplitude waveforms) exhibited specific clusters based on each period of threat exposure [67].

In the analysis of the EEG signals, for each channel $k$, all the PSDs were normalized according to $P S D_{n}^{C H_{k}}\left(w_{i}\right)=\operatorname{PSD}\left(w_{i}\right) / \sum_{j=1}^{w_{\max }} P S D\left(w_{j}\right)$, where $\left\{w_{i}\right\}$ corresponds to all frequencies. Therefore, the PSD can be interpreted as empirical probability distributions, where the normalized power 
represents the probability of detecting an activation in a given interval of frequencies. For statistical evaluations of the signal powers comparing the initial and final sessions of the training protocol, we considered the ratio $r$ calculated from the normalized power of the last session over the normalized power of the first session. Then, we calculated the median ratio of the $n=7$ participants and their respective confidence intervals (CIs, 95\%). The ratios were calculated considering each subject at each channel of each frequency band: theta $(4-8 \mathrm{~Hz})$, alpha $(8-12 \mathrm{~Hz})$, beta $(12-30 \mathrm{~Hz})$ and gamma (30-45 $\mathrm{Hz})[58]$.

Regarding the CCE calculations, the only data that were included were from correct executions, while times greater than $1500 \mathrm{~ms}$ and less than $200 \mathrm{~ms}$ were excluded (3.4\% of all trials) [28]. Statistical comparisons were performed between CCE averages while considering that the visual and tactile stimuli were applied on the same side (SS) and on the opposite side (OS). The prior visualization of the virtual prosthesis movements with and without associated vibrotactile stimulation were both considered statistical factors in the initial and final sessions. Thus, a two-way ANOVA with Tukey-Kramer post hoc correction was applied to compare the CCE means for the stimuli that were applied on the same side and opposite side. To describe the degree of freedom of two-way $F$-statistics, we adopted the following notation: $\mathrm{F}$ (factor $\mathrm{A}:(a-1)$, factor $\mathrm{B}:(b-1)$, interaction $\mathrm{A} * \mathrm{~B}:(a-1)(b-1)$, general error: $a b(n-1))$, where $a$ : number of groups in factor $\mathrm{A} ; b$ : number of groups in factor $\mathrm{B}$; and $n$ : total number of samples.

To evaluate the relationships between CCE and SCR measures, Pearson's correlation coefficients were calculated together with the description of the respective linear regressions for each variable pair. Only the coefficients with $p<\alpha$, tested with a t-test, were considered significant. The analysis related to the sense of ownership and agency was included using the absolute values reported by each of the participants (from 0 to 10) at the beginning and end of the protocol.

To analyze performance during motor control tasks, across training with the EMG-based HMI and the test conducted to investigate the perception of virtual prosthesis movements using vibrotactile stimuli, the following variables were analyzed: a) execution time and b) success rate. Only correct execution attempts were considered for (a), and all attempts were considered for (b). The target angles were separated into intermediate $\left(30^{\circ}\right.$ and $\left.60^{\circ}\right)$ and extreme $\left(0^{\circ}\right.$ and $\left.90^{\circ}\right)$ angles. In addition, the data were grouped into three difficulty levels related to the precision of movements, i.e., $15^{\circ}, 10^{\circ}$ and $5^{\circ}$ to the target angle.

The task execution times were compared among these three difficulty levels while separately considering the intermediate and extreme target angles (Kruskal-Wallis test followed by TukeyKramer post hoc correction). Comparisons among execution times, while considering the intermediate and extreme target angles, were also performed (using the Mann-Whitney test), as well as the analysis 
of success rates by comparing among average proportions and CIs (95\%). In association with these tests, by using the logistic regression technique [68], it was possible to estimate how much the task execution times during the training protocol with EMG-based HMI (visual and vibrotactile stimulation) had information about the performance during the perception evaluation test of virtual prosthesis movements using vibrotactile stimuli without the aid of vision and vice versa. Due to limitations of the vibrotactile stimulation device, with activation of the vibrating motors by rows and peaks of vibration intensity at angles of $0^{\circ}, 30^{\circ}, 60^{\circ}$ and $90^{\circ}$, the target angle of $45^{\circ}$ used during the preliminary stage of the training protocol was not considered in this analysis. This angle did not introduce any difference in terms of performance in comparison to the other intermediate angles $\left(30^{\circ}\right.$ and $60^{\circ}$ ) (see Additional file 4); that is, it did not compromise the findings and interpretations.

\section{Results}

The training protocol induced and enhanced virtual prosthesis embodiment, in addition to providing an improvement in the motor control of the stump muscles. Furthermore, the participants were able to guide the movements of the virtual prosthesis using vibrotactile stimuli without the aid of vision.

\section{Virtual prosthesis embodiment}

Virtual prosthesis embodiment and enhancement through the training protocol using EMGbased HMI was consistently observed in different analyses:

a) Affective measurement - all participants reacted affectively to a threat to the virtual prosthesis, indicated by a significant increase in $\operatorname{SCR}(\mathrm{F}(1,1,1,1437)=53.3$, p (before/after $)=1.084 \times 10^{-}$ 13; $\mathrm{F}(1,1,1,1437)=85.15$, $\mathrm{p}$ (initial/final $)=9.55 \times 10^{-20}$; initial session post hoc $\mathrm{p}$ (initial-before, initialafter),(initial-before, final-before),(initial-before, final-after) $=0.000012$; final session post hoc $\mathrm{p}$ (initial-after, final-after),(final-before, final-after)=0.0001) (Figure 4-A).

b) Spatial perception measurement - the significant increase in the CCE values obtained when the stimuli were applied on the same side of the body than on the opposite sides at the end of the training, indicating that the visual stimuli applied in the virtual environment were considered close to the real body. That is, there was a recalibration of the peripersonal space to include the virtual prosthesis (initial: $\mathrm{F}(1,1,1,25)=0.0798, \quad \mathrm{p}(\mathrm{VR}-\mathrm{VT} / \mathrm{VR} \quad$ only $)=0.7798 ; \mathrm{F}(1,1,1,25)=0.0668$, $\mathrm{p}(\mathrm{SS}, \mathrm{OS})=0.7982$; and final: $\mathrm{F}(1,1,1,25)=0.0133, \mathrm{p}(\mathrm{VT} / \mathrm{VR}$ only $)=0.9091 ; \mathrm{F}(1,1,1,25)=7.7396$, 
$\mathrm{p}(\mathrm{SS}, \mathrm{OS})=0.0101$; post hoc $\mathrm{p}(\mathrm{VR}-\mathrm{VT}: \mathrm{SS}, \mathrm{VR}-\mathrm{VT}: \mathrm{OP})=0.0467, \mathrm{p}(\mathrm{VR}$ only: SS, VR only: OP $)=0.0466)$ (Figure 4-B).

c) Motor measurement - motor training with the EMG-based HMI provided an improvement in the ability to control the virtual prosthesis, considering that there was a success rate $>75 \%$, even with the progressive increase in the difficulty of the tasks (Figure 6-A.3).

d) High self-perception regarding the sense of ownership and agency of the virtual prosthesis by most participants from the beginning of the training, with scores $\geq 7$. In most cases, these scores were increased or maintained throughout the protocol, except for two participants, "C" and "D", who reported a decrease in the sense of agency at the end of the training (Figure 4-C). These results associated with qualitative reports (see Additional file 1) showed that EMG-based HMI training induced the participants to self-perceive that the virtual prosthesis was part of their own body and that they could voluntarily control it.

e) Significant changes in resting-state brain rhythms at the end of the training in comparison to those in the beginning. These changes were observed on specific electrodes: theta activity decreased at the F8 and CZ electrodes; alpha activity increased at P3, P8 and PZ and decreased at CP1; beta activity increased at FZ and P7; and gamma activity decreased at the PZ, P3, O1, and C1 electrodes (Figure 5).

The increase in SCR amplitude occurred from the beginning of the sessions. At the end of the training, this increase was significantly greater in magnitude than at the initial evaluation (Figure 4A). There was a significant difference in CCE responses only at the end of the training and no difference at the beginning (Figure 4-B). However, there was a significant correlation between the mean SCR and CCE values (stimuli applied on the same side) (initial $r=0.82, \mathrm{p}=0.0472$; final $r=0.90$, $\mathrm{p}=0.0142$ ) at the beginning and end of the training (Figure 4-D). 
A

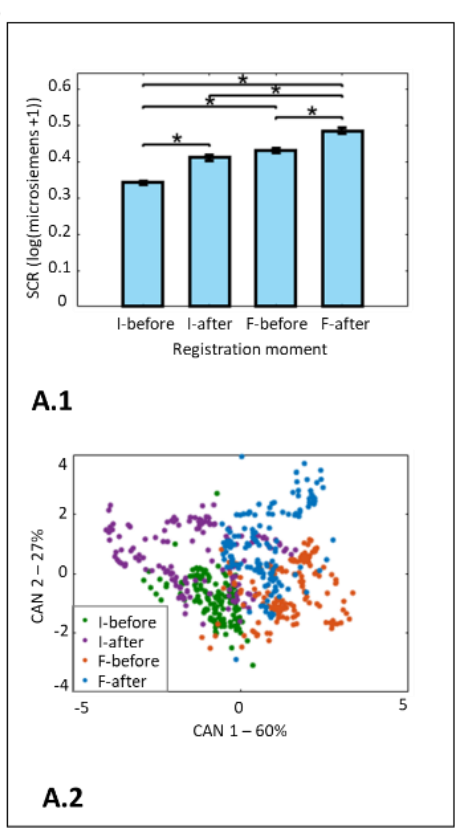

B

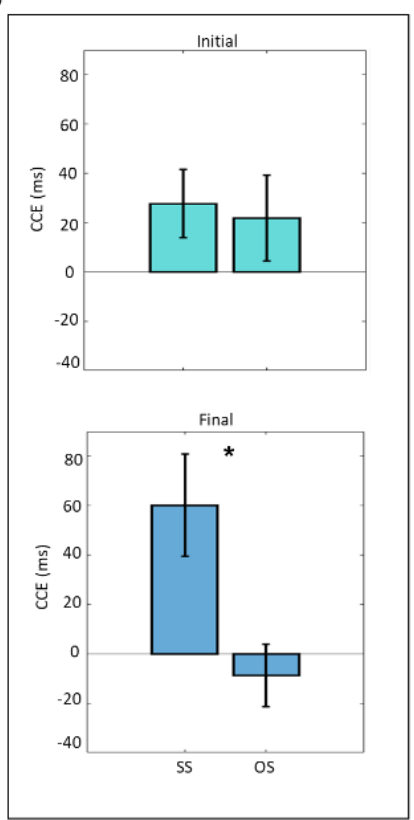

C

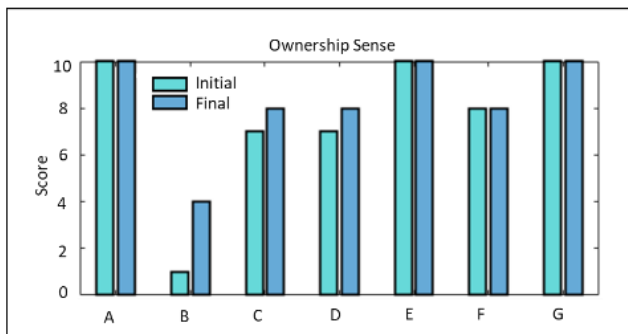

C.1

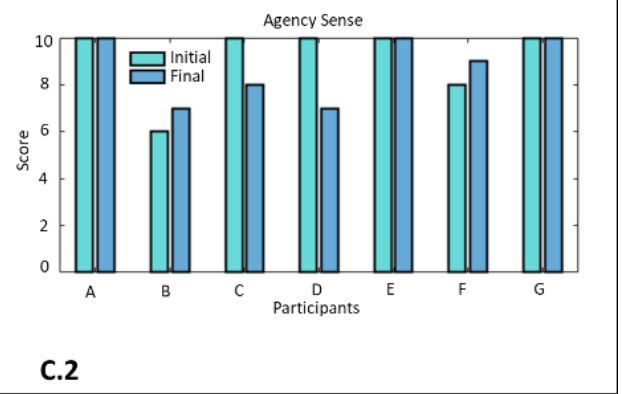

D
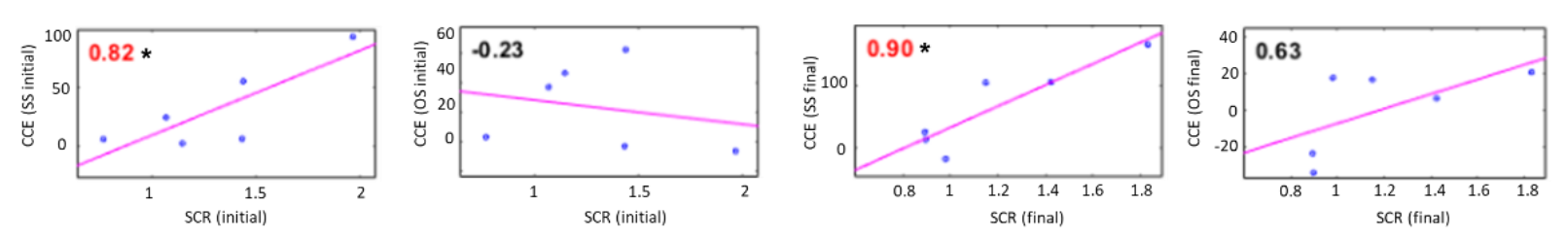

Figure 4 - Training induces and improves virtual prosthesis embodiment. A) Skin conductance response (SCR) to a threat to the virtual prosthesis. A.1) Two-way ANOVA with Tukey-Kramer correction. A.2) Application of one-way MANOVA followed by canonical discriminant analysis. B) Crossmodal congruency task (CCT) and crossmodal congruency effect (CCE) (two-way ANOVA with Tukey-Kramer correction). Comparison for stimuli applied on the same side (SS) and opposite side (OS). C) Self-perception (absolute values quantified by the participants). C.1) Sense of ownership. C.2) Sense of agency. D) Correlations between the SCR and CCE results (Pearson's correlation coefficients). $* \mathrm{p}<0.05$. 

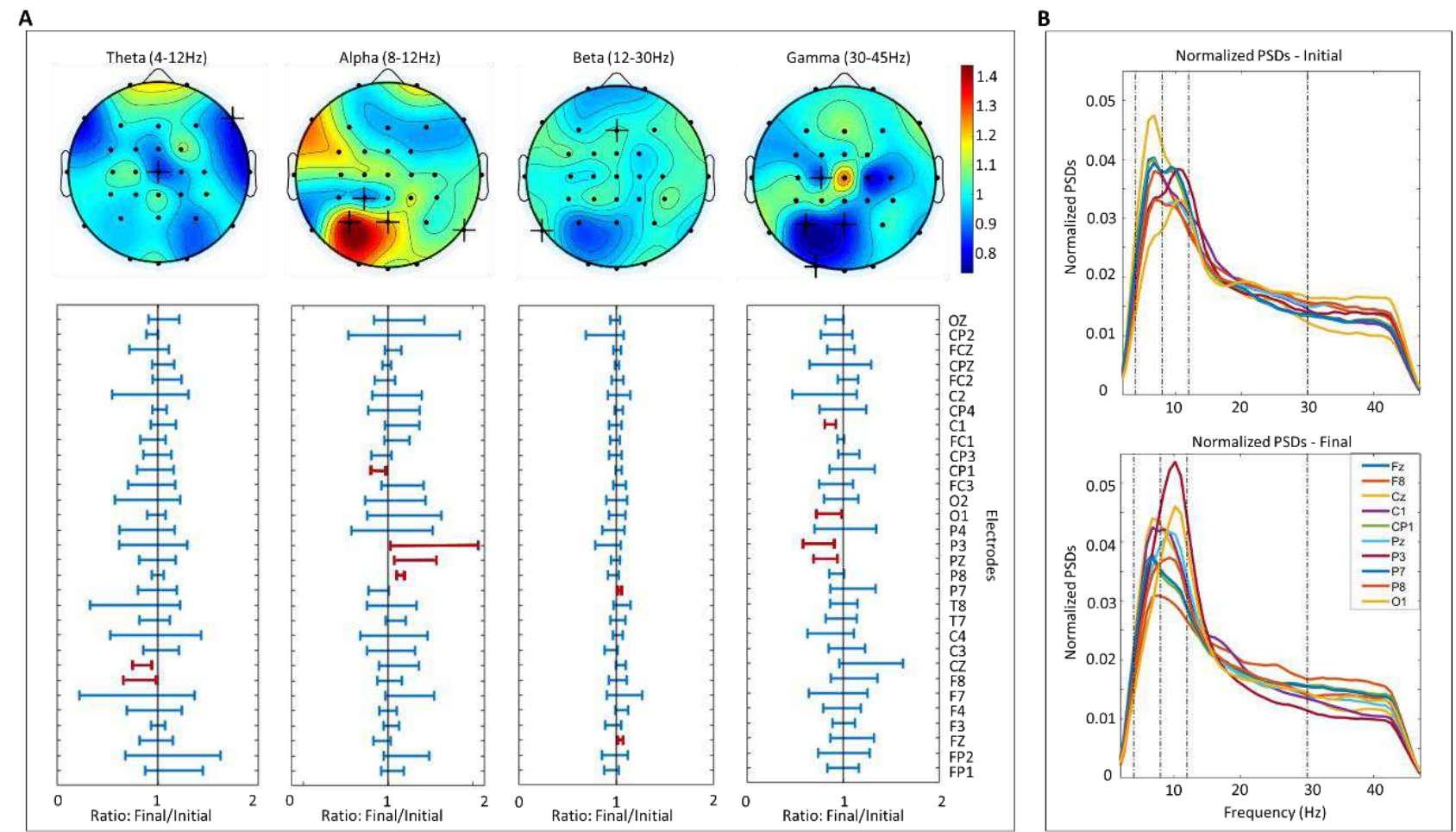

Figure 5 - Changes in resting-state brain rhythms. A) Topoplots of the normalized power ratio considering the final and initial sessions related to theta, alpha, beta and gamma frequency bands. The symbol ' + ' highlights statistical significances. Below the topoplots are the ratio medians ( $n=7$ subjects) with their respective CIs calculated from the normalized powers, considering the final over the initial sessions for each electrode. Electrodes with significant changes are highlighted in red. B) Normalized PSDs in the initial and final sessions of the channels: FZ, F8, CZ, C1, CP1, PZ, P3, P7, P8 and O1.

\section{Improvement of the motor control}

Training using an EMG-based HMI was critical to improve virtual prosthesis embodiment and motor control training of the amputated limb. Motor training resulted in an increase in the subjects' ability to control the virtual prosthesis considering that there was a high success rate $(>75 \%)$ even with progressive increases in task difficulty (Figure 6-C and Additional file 5). However, although the success rate was always high, the execution time was longer in the more difficult/complex conditions. With intermediate angles $\left(30^{\circ}\right.$ and $\left.60^{\circ}\right)$, the time to execute the tasks was longer than with extreme target angles $\left(0^{\circ}\right.$ and $\left.90^{\circ}\right)$ regardless of the precision of movement required during the task (precision of movement $15^{\circ}: U=263, p=0.0063 ; 10^{\circ}: U=288, p=1.0335 \times 10^{-4} ; 5^{\circ}: U=301, p=7.4678 \times 10^{-6}$ ) (Figure 6-A). The difficulty associated with greater movement precision $\left(5^{\circ}\right.$ range in relation to the target angle) also demanded significantly more time for task execution $\left(\mathrm{H}=18.038, \mathrm{p}=1.2109 \times 10^{-4}\right.$; post hoc $\left.\mathrm{p}\left(5^{\circ}, 15^{\circ}\right)=0.0001 ; \mathrm{p}\left(5^{\circ}, 10^{\circ}\right)=0.0192\right)$ (Figure 6-B). 
A
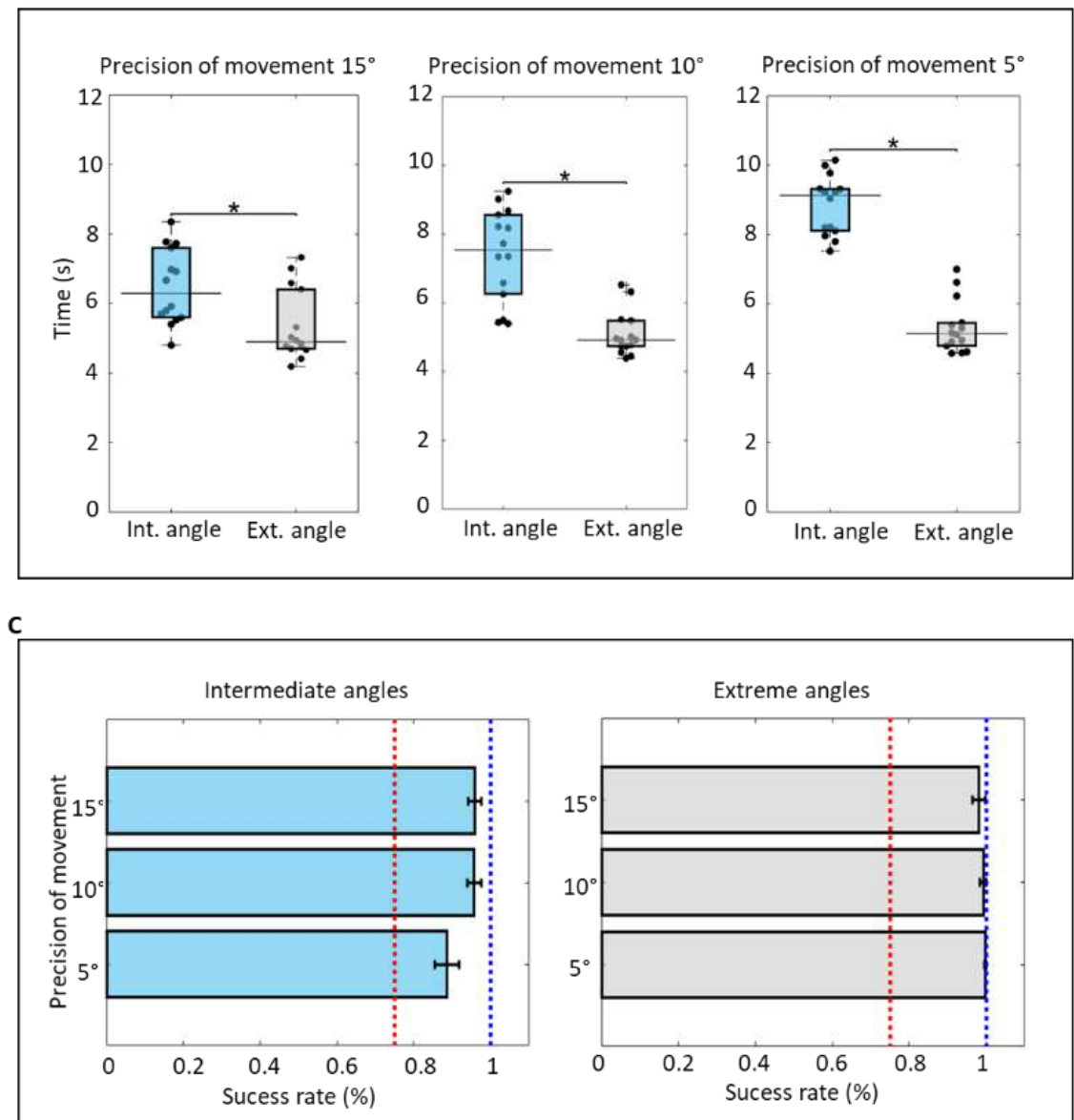

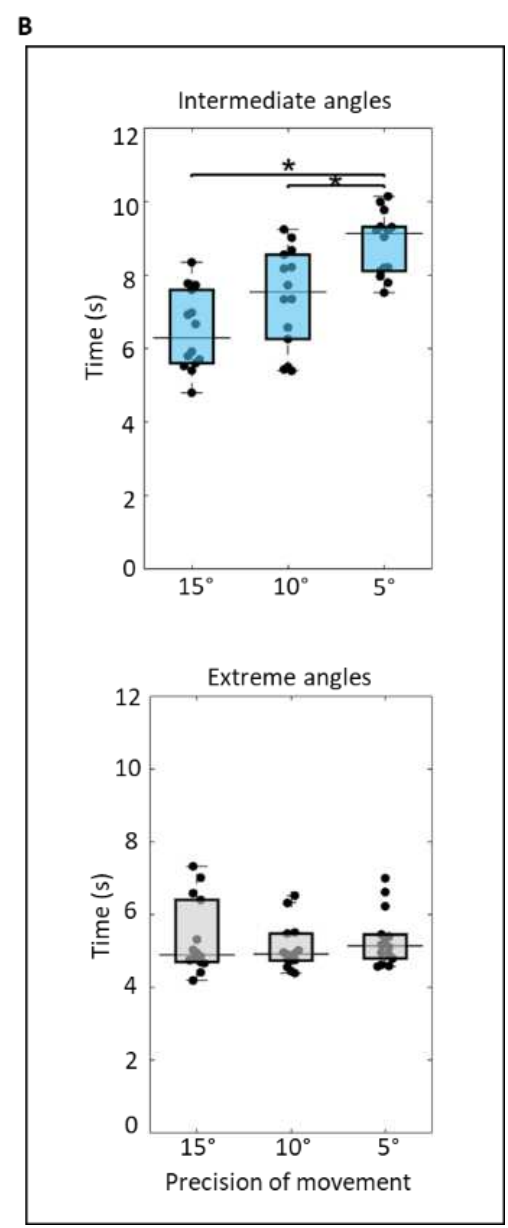

Figure 6 - Performance. A) Training with the EMG-based HMI (visual and vibrotactile stimulation). A.1) Comparison of execution times between the intermediate $\left(30^{\circ}\right.$ and $\left.60^{\circ}\right)$ and extreme $\left(0^{\circ}\right.$ and $\left.90^{\circ}\right)$ target angles (Mann-Whitney test). A.2) Comparison of execution times across levels of difficulty related to the precision of movement $\left(15^{\circ}, 10^{\circ}\right.$ and $5^{\circ}$ of variation in relation to the target angle) for the intermediate and extreme angles (Kruskal-Wallis with Tukey-Kramer correction). A.3) Success rate on tasks involving intermediate and extreme target angles at each level of required movement precision (average of the proportion and CI). The red line indicates a success rate of $75 \%$, and the blue line indicates $100 \%$.

\section{Perception of virtual prosthesis movements using vibrotactile stimuli}

The subjects' performance in controlling the virtual prosthesis was also tested using vibrotactile stimuli without visual stimulation. The results showed that the participants were able to associate the patterns of vibrotactile stimulation applied on their back with the movements of the virtual prosthesis, allowing them to guide their movements without the aid of vision. As expected, when the control of the movements required more precision, such as during the tasks involving the intermediate target angles, the participants required a significantly longer time for execution than with the extreme angles. This effect occurred at all levels of difficulty with respect to precision of movement (precision of movement $15^{\circ}$ : $U=301, p=7.4678 \times 10^{-6} ; 10^{\circ}: U=293, p=3.9171 \times 10^{-5} ; 5^{\circ}: U=293$, 
$\left.\mathrm{p}=3.9171 \times 10^{-5}\right)($ Figure $7-\mathrm{A})$. When analyzing the intermediate and extreme target angles separately, no significant differences in the execution times were found across the three precision of movement levels (Figure 7-B). However, despite the high success rate involving the extreme angles regardless of the level of difficulty, for the intermediate target angles, when there was a need for greater precision in movement (range of $5^{\circ}$ in relation to the target angle), the success rate was low $(<75 \%)$ (Figure 7 $\mathrm{C}$ and Additional file 6).

From the classification analyses using multivariate logistic regression (logit) and taking all the execution time samples into consideration, two scenarios were evaluated: a) using the task execution time obtained during the EMG-based HMI training (visual and vibrotactile stimulation) as the basis for the logistic classifier to estimate the angles reproduced during the perception evaluation test of virtual prosthesis movements using vibrotactile stimuli without the aid of vision and b) using the execution time obtained from the virtual prosthesis movements using vibrotactile stimuli without the aid of vision as a basis for the logistic classifier to estimate the angles reproduced during the motor control training (visual and vibrotactile stimulation).

In scenario (a), the target angle $0^{\circ}$ was recognized with $84 \%$ probability as the angle $90^{\circ}$. However, the logit model was able to correctly identify, with $89 \%$ probability, the $90^{\circ}$ target. With the intermediate targets, the model correctly identified the $30^{\circ}$ target angle with $59 \%$ probability and the $60^{\circ}$ angle with $31 \%$ probability. Although there was a high misclassification rate between these two angles, the $60^{\circ}$ target angle was classified as $30^{\circ}$ with a probability of $41 \%$ and the $30^{\circ}$ angle as $60^{\circ}$ with a probability of $21 \%$ (Figure $8-\mathrm{A}$ ).

In scenario (b), a high level of distinction was observed between the extreme target angles and the intermediate angles, with low specificity between each of the four executed angles. Although there was low accuracy in classifying the extreme target angles $\left(0^{\circ}\right.$ was correctly recognized with $20 \%$ probability and $90^{\circ}$ with $24 \%$ probability) and there was no specific identification between the intermediate target angles $\left(30^{\circ}\right.$ correctly recognized with $48 \%$ probability and $60^{\circ}$ with $47 \%$ probability), the classifier was able to distinguish the intermediate angles from the extreme angles with high accuracy (Figure 8-B). 
A

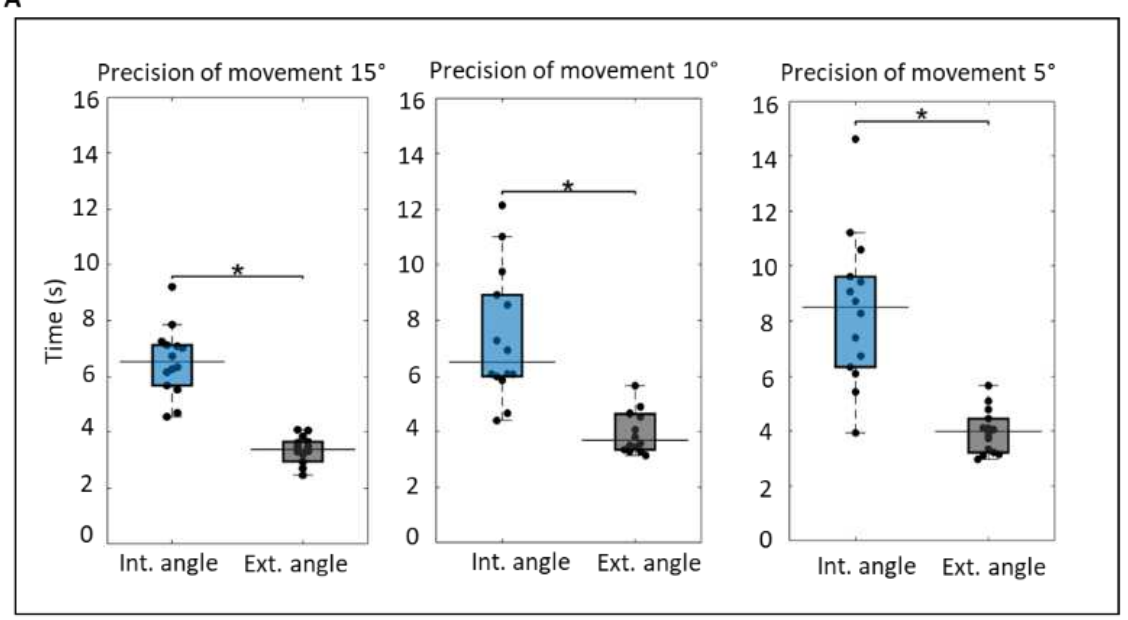

c

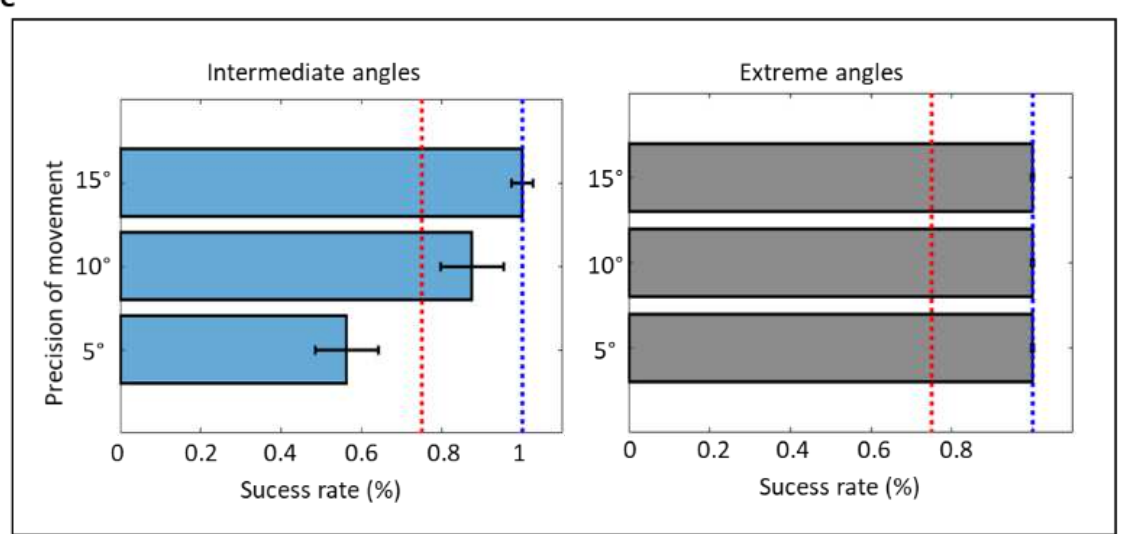

B

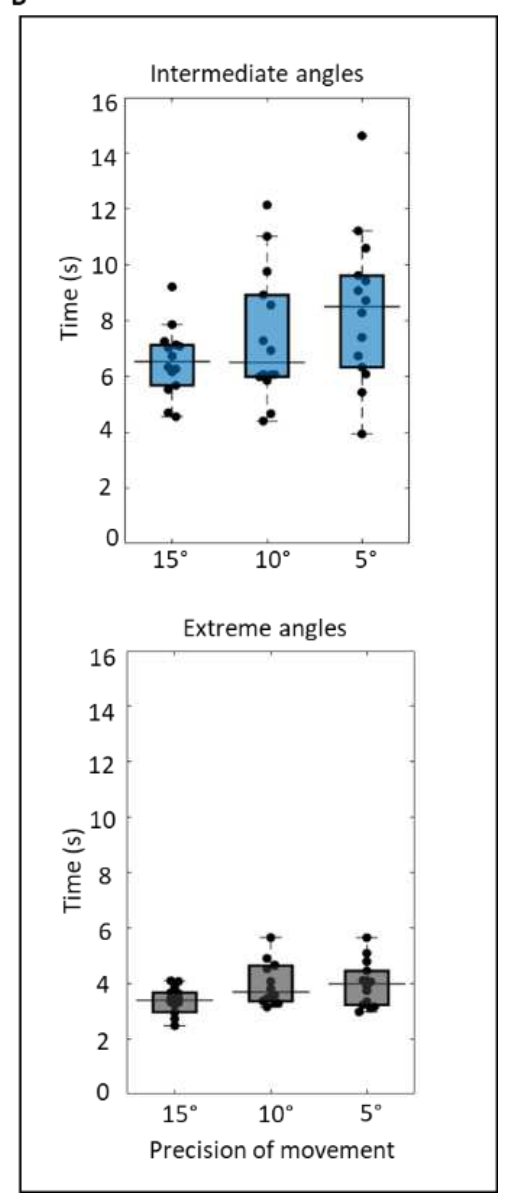

Figure 7 - Performance. Test to assess the perception of virtual prosthesis movements using vibrotactile stimuli without vision. B.1) Comparison of execution times between the intermediate $\left(30^{\circ}\right.$ and $\left.60^{\circ}\right)$ and extreme $\left(0^{\circ}\right.$ and $\left.90^{\circ}\right)$ target angles (Mann-Whitney test). B.2) Comparison of execution times across levels of difficulty related to the precision of movement $\left(15^{\circ}, 10^{\circ}\right.$ and $5^{\circ}$ of variation in relation to the target angle) for the intermediate and extreme angles (Kruskal-Wallis with TukeyKramer correction). B.3) Success rate on tasks involving intermediate and extreme target angles at each level of required movement precision (average of the proportion and $\mathrm{CI}$ ). The red line indicates a success rate of $75 \%$, and the blue line indicates $100 \%$. ${ }^{*} p<0.05$. C) Logistic regression analysis. C.1) Estimation of how much the execution times during the training protocol with the EMG-based HMI (visual and vibrotactile stimulation) can classify performance during the perception evaluation test of the virtual prosthesis movements using vibrotactile stimuli without vision. C.2) Estimation of how much the execution times during the perception evaluation test of the virtual prosthesis movement using vibrotactile stimuli without vision can classify the performance during the training protocol with the EMG-based HMI (visual and vibrotactile stimulation). 


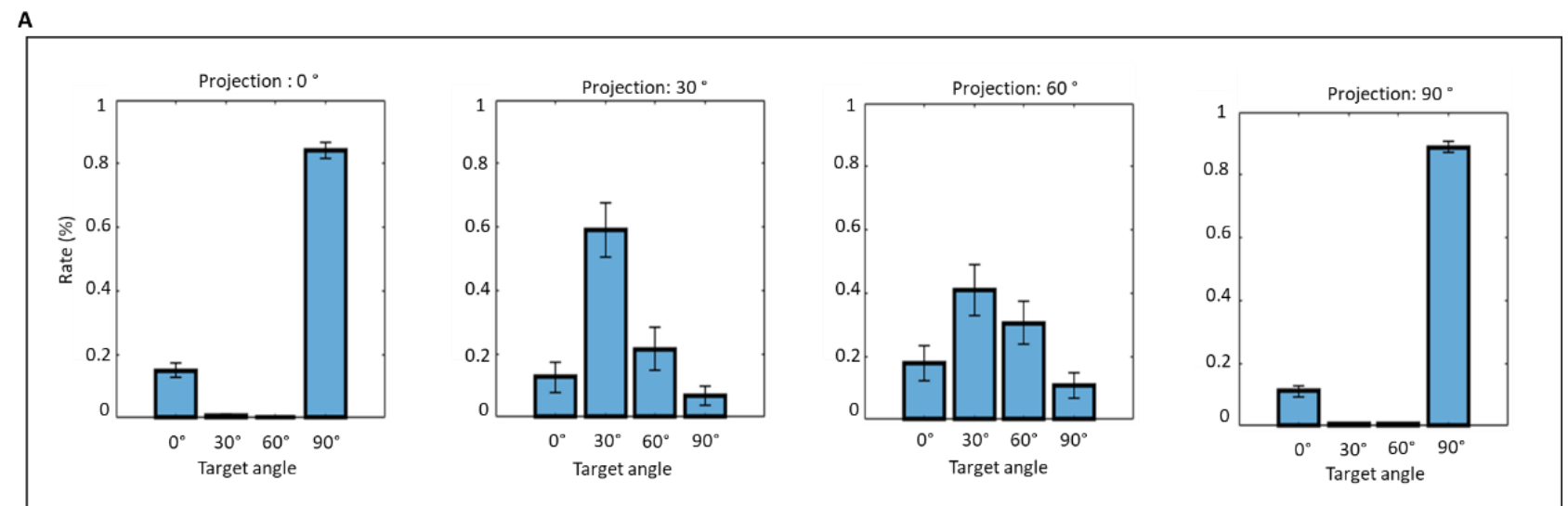

B

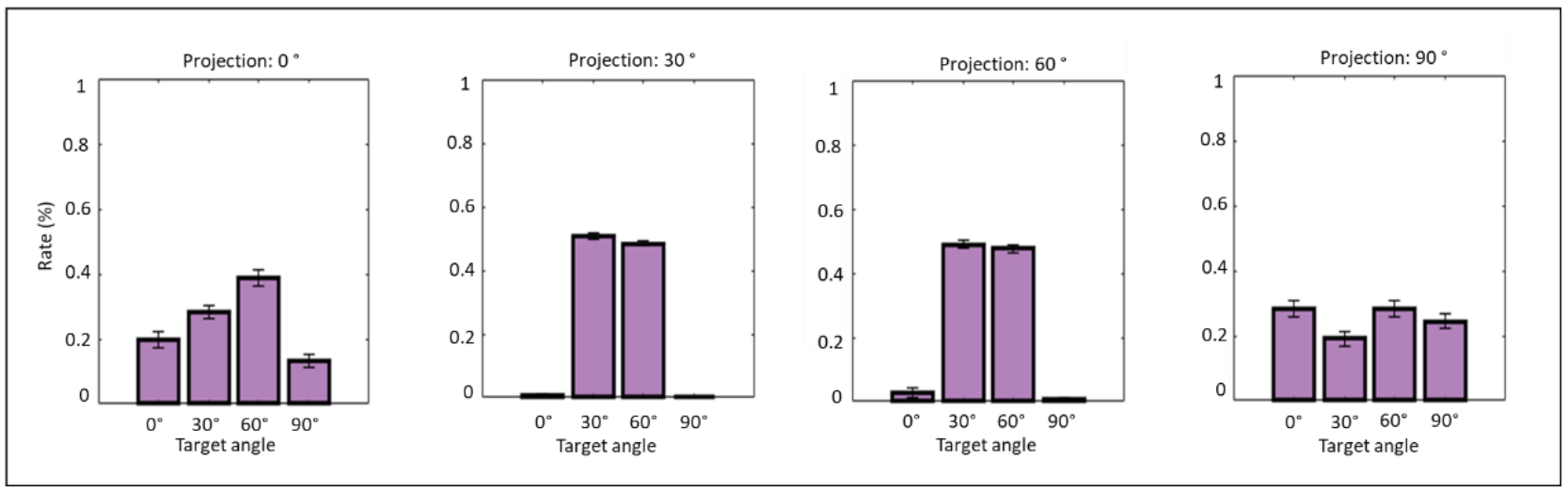

Figure 8 - Performance. Logistic regression analysis. C.1) Estimation of how much the execution times during the training protocol with the EMG-based HMI (visual and vibrotactile stimulation) can classify performance during the perception evaluation test of the virtual prosthesis movements using vibrotactile stimuli without vision. C.2) Estimation of how much the execution times during the perception evaluation test of the virtual prosthesis movement using vibrotactile stimuli without vision can classify the performance during the training protocol with the EMG-based HMI (visual and vibrotactile stimulation).

\section{Discussion}

The experimental protocol used in this study was able to induce and enhance embodiment of a virtual prosthesis in people with transfemoral amputation. In addition, better motor control associated with the stump muscles was observed. We also identified changes in resting-state brain activity patterns, suggesting neuroplasticity throughout the training sessions. Furthermore, the participants were able to guide virtual prosthesis movements using vibrotactile stimuli without the aid of vision, showing that these stimuli can be used as an alternative sensorial path to facilitate movement perception.

\section{Virtual prosthesis embodiment}


In summary, in relation to the virtual prosthesis embodiment, we observed that the affective response was immediate, but with training, there was an amplification of this response. These findings, along with the recalibration of the peripersonal space and correlation between these responses and the increased control capacity with training, showed an improvement in the embodiment over time. The high indices of self-perception declared by the subjects regarding their sense of ownership and agency over the virtual prosthesis and the changes in resting-state EEG activity also corroborated it.

The immediate affective response can be explained by visual and proprioceptive congruence of the real and virtual body experienced through the first-person perspective, which is not dependent on visuomotor or visuotactile stimulation [69-70]. The recalibration of the peripersonal space, which occurred only at the end of the training, may be linked to the processing of body perception depending on learning motor skills acquired during the training sessions [56, 71-72]. The increase in the ability to control the virtual prosthesis during training indicated that the participants were able to use visual and vibrotactile feedback for motor planning and execution in the control of virtual prosthesis movements [10].

Participants also reported high self-perception that the virtual prosthesis was part of their own body and that they could voluntarily control it [62-63]. This perception remained stable or increased over the course of the training in most cases. Only two participants reported a decreased sense of agency at the end of the protocol. However, for both of them, the score given in the initial evaluation for the agency sense was already the maximum value. Most likely, this result was related to the expectations created by these participants that control would be easier throughout the sessions, which did not occur due to the progressive increase in the difficulty imposed during training. Additionally, it is worth noting that this effect did not affect their sense of ownership because reports from both individuals increased at the end of the training, which reinforces this interpretation.

In relation to the study of the patterns of resting-state EEG activities, we found significant changes at the end of the training in records made on the prefrontal, frontal, parietal and occipital regions [73]. The attenuation of theta activity identified in the prefrontal cortex could indicate permanent neural changes associated with the cognitive effort and concentration required during the training [74-75]. In addition, changes in the theta, beta and gamma oscillations detected in central front electrodes corresponding to brain areas responsible for motor processing [73] suggested adaptations in cognitive [75-77] and motor processing [78].

We also observed changes in alpha, beta and gamma rhythms in recordings on the posterior parietal cortex (PPC). The wide changes in electrophysiological activity in this region pointed to neural adaptations associated with sensory integration both to guide action and to build body perception [7884]. Moreover, we identified an attenuation of the gamma rhythm on the occipital cortex (OC), which 
may also be involved in sensory processing, considering that the OC is interconnected with the PPC and with the temporal cortex, yielding important pathways for visual orientation and object recognition [48,81-82]. Other studies have also described that the frontoparietal network, involving the PPC and premotor cortex, important regions for processing peripersonal space representation $[79,85]$, is also related to the development of ownership and/or agency sense over an external object [86-90].

These findings together suggested that functional changes in the patterns of resting-state EEG activities can reflect neural adaptations linked to sensorimotor processing and body perception, thereby contributing to the development and enhancement of virtual prosthesis embodiment as well as motor learning.

To our knowledge, this is the first clinical study that investigates the embodiment of a virtual prosthesis, covering different aspects such as physiological and behavioral responses, in addition to the participants' own reports. The results found are promising and open the way for future studies in the areas of cognitive neuroscience and clinical application.

\section{Improvement of the motor control}

Performance analyses during the training using an EMG-based HMI indicated that there was an improvement in the ability to control the virtual prosthesis due to a motor learning process [57,91]. The time required for the participants to perform the tasks was longer for the intermediate target angles than for the extremities. For the intermediate angles, the time was even greater when the task required greater precision. The differences in these times can be explained by the level of complexity of the motor control strategies for the realization of each one: simpler strategies in the case of extremity angles and more complex strategies for reproducing intermediate target angles, especially in more precise tasks.

This interpretation can be supported by motor control theories based on feedforward and feedback mechanisms [92-93]. Thus, in conditions where movement strategies were simpler, as in the tasks with extreme target angles, motor control occurred largely through feedforward mechanisms from the estimation of sensory consequences using copies of the efferent motor commands. In this way, the execution times were shorter because the predicted movements were similar to real movements, without the need for major corrections during the execution. However, during tasks with more complex motor control strategies, those with intermediate target angles and higher precision requirements, motor control occurred mainly from sensory feedback by comparing predicted and actual movements [91-93]. In these cases, corrections and adjustments of the movement in real time were determinant and explained the longer execution times during these tasks. 
Training protocols with EMG-based HMI have already been used in other clinical contexts [15-16, 94], but there are still few studies on people with amputation involving immersion in VR environments. In view of the results presented here, this system can also be used as a therapeutic resource for motor control training.

\section{Perception of virtual prosthesis movements using vibrotactile stimuli}

At the end of the training sessions, a test was performed to evaluate how and whether vibrotactile stimuli could facilitate the perception of virtual prosthesis movements without a visual aid. All participants were able to guide the virtual prosthesis movements by using vibrotactile stimulation as a reference. By analyzing participant performance during this test, a pattern of execution and motor control similar to that found during the training (visual and vibrotactile stimulations) was identified: longer execution times during tasks involving the intermediate target angles and shorter execution times during the tasks involving the extreme target angles. However, in this test, the level of movement precision was not a determinant of the execution time.

The success rate during the execution of tasks involving extreme target angles was high for all levels of movement precision. However, for intermediate angles, although the success rate was high in conditions with lower movement accuracy, the participants achieved a lower success rate in conditions with high levels of difficulty. These results showed that for tasks requiring more complexity in the motor control strategies (intermediate angles and greater precision), the visual aid was critical to produce appropriate movement.

Similar performances in the execution times during the tasks in this test (vibrotactile stimulation without the aid of vision) and the training protocol (visual and vibrotactile stimulation) indicated a process of motor learning that was maintained even when the visual afferences were removed. The correlations evidenced by the logistic regression analyses corroborated this interpretation, suggesting that the participants adopted the same motor control strategies in both protocols.

Thus, the high success rate with a shorter time to execute the extreme angles may be related to the updating of internal models and to the improvement in movement prediction, which does not require many corrections during execution [91, 93]. Moreover, in conditions requiring more complex motor strategies, e.g., to reach intermediate target angles, motor control was again more dependent on real-time corrections based on sensory feedback [92-93]. Mainly, the tasks that demanded higher precision, where the success rate was low, showed how much the visual stimulus was determinant for performing and correcting errors during these movements. Therefore, we can conclude that vibratory stimulation facilitated the perception of virtual prosthesis movements, but there is a limit regarding 
motor actions with higher levels of complexity and precision; in these cases, vision played a crucial role.

The implications of these results are relevant both in the development of new therapeutic approaches and in the field of neuroprostheses, showing that the use of vibrotactile stimulation provides a viable alternative to facilitate the perception of movements.

\section{Limitations}

Although all variables evaluated in this protocol were based on the interaction of the participant with the virtual prosthesis and completely different from those performed during regular rehabilitation, the possible contributions from the multidisciplinary treatment carried out concurrently on the results found cannot be excluded.

Future studies with a larger sample and control groups, in addition to randomized clinical trials, are still necessary. Studies with follow-up are also indicated for a better understanding of whether the modifications are permanent and can be extended to the use of physical prostheses.

\section{Conclusion}

This study showed that by using an EMG-based HMI that integrated visual and vibrotactile feedback to the volitional control of a virtual prosthesis associated with a set of directed and progressive tasks, it is possible to induce and enhance virtual prosthesis embodiment. The protocol proposed here also provided training of the stump muscles involved in the control of virtual prosthesis movements. These results show that the use of an EMG-based HMI associated with interactive training is a viable option to study and induce embodiment of a virtual prosthetic limb. In addition, it can be used as a therapeutic protocol for motor control training and to assist during the process of adaptation to and acceptance of the use of prostheses. Finally, the vibrotactile stimuli facilitated the perception of virtual prosthesis movements without vision aid, indicating that the use of this simple, noninvasive, and low-cost method is a viable alternative to be implemented in the development of neuroprostheses and new therapeutic strategies.

\section{List of abbreviations}

CCE - crossmodal congruency effect

CCT - crossmodal congruency task 
EEG - electroencephalography

EMG - electromyography

HMI - human-machine interface

MVIC - maximum voluntary isometric contraction

OC - occipital cortex

PPC - posterior parietal cortex

PSD - power spectral density

RMS - root mean square

SCR - skin conductance response

VR - virtual reality

\section{Declarations}

Ethics approval and consent to participate

The research protocol was approved by the Ethics and Research Committee of the Universidade Federal de São Paulo ( ${ }^{\circ}$ 3.030.942) and of the Hospital Municipal Dr. José de Carvalho Florence $\left(\mathrm{n}^{\circ} 3.273 .170\right)$, Brazil. The participants provided written consent prior to the start of the study, and all ethical recommendations and regulations were followed.

\section{Consent for publication}

Consent to use the image was obtained from all participants.

\section{Competing interests}

The authors declare that they have no competing interests.

\section{Funding}

This study was funded by Conselho Nacional de Desenvolvimento Científico e Tecnológico ( ${ }^{\circ}$ 442563-2016/7) and Coordenação de Aperfeiçoamento de Pessoal de Nível Superior.

\section{Acknowledgments}

We thank all the volunteers who participated in this research. We also thank Dra. Karina Rabello Casali, Dr. Henrique Alves Amorim, Dr. Adenauer Girardi Casali, Dr. Matheus Cardoso Moraes (Universidade Federal de São Paulo - Instituto de Ciência e Tecnologia), Fabiano Vittoretti Pereira, Renato José Soares, and all collaborators of the Centro de Reabilitação Lucy Montoro and Associação 
Paulista para o Desenvolvimento da Medicina (SPDM) for the whole support during the experiments. Illustration A.1 in Figure 1 was designed by Kjpargeter/Freepik.

\section{References}

1 Unwin N. Epidemiology of lower extremity amputation in centres in Europe, North America and East Asia. Br J Surg. 2000. doi: 10.1046/j.1365-2168.2000.01344.x.

2 Webster JB. Lower Limb Amputation Care Across the Active Duty Military and Veteran Populations. Phys Med Rehabil Clin N Am. 2019. doi: 10.1016/j.pmr.2018.08.008.

3 Biddiss EA, Chau TT. Upper limb prosthesis use and abandonment: a survey of the last 25 years. Prosthet Orthot Int. 2007. doi: 10.1080/03093640600994581.

4 Jang CH, Yang HS, Yang HE, Lee SY, Kwon JW, Yun BD, Choi JY, Kim SN, Jeong HW. A survey on activities of daily living and occupations of upper extremity amputees. Ann Rehabil Med. 2011. doi: 10.5535/arm.2011.35.6.907.

5 Gailey R, McFarland LV, Cooper RA, Czerniecki J, Gambel JM, Hubbard S, Maynard C, Smith DG, Raya M, Reiber GE. Unilateral lower-limb loss: prosthetic device use and functional outcomes in servicemembers from Vietnam war and OIF/OEF conflicts. J Rehabil Res Dev. 2010. doi: 10.1682/jrrd.2009.04.0039.

6 Østlie K, Lesjø IM, Franklin RJ, Garfelt B, Skjeldal OH, Magnus P. Prosthesis rejection in acquired major upper-limb amputees: a population-based survey. Disabil Rehabil Assist Technol. 2012. doi: 10.3109/17483107.2011.635405.

7 Atherton R, Robertson N. Psychological adjustment to lower limb amputation amongst prosthesis users. Disabil Rehabil. 2006. doi: 10.1080/09638280600551674.

8 Petrini FM, Valle G, Bumbasirevic M, Barberi F, Bortolotti D, Cvancara P, Hiairrassary A, Mijovic P, Sverrisson AÖ, Pedrocchi A, Divoux JL, Popovic I, Lechler K, Mijovic B, Guiraud D, Stieglitz T, Alexandersson A, Micera S, Lesic A, Raspopovic S. Enhancing functional abilities and cognitive integration of the lower limb prosthesis. Sci Transl Med. 2019. doi: 10.1126/scitranslmed.aav8939.

9 Makin, T. R., de Vignemont, F., \& Faisal, A. A. Neurocognitive barriers to the embodiment of technology. Nature Biomedical Engineering. 2017. doi: 10.1038/s41551-016-0014.

10 de Vignemont F. Embodiment, ownership and disownership. Conscious Cogn. 2011. doi: 10.1016/j.concog.2010.09.004.

11 Hellman RB, Chang E, Tanner J, Helms Tillery SI, Santos VJ. A robot hand testbed designed for enhancing embodiment and functional neurorehabilitation of body schema in subjects with upper limb impairment or loss. Front Hum Neurosci. 2015. doi: 10.3389/fnhum.2015.00026.

12 Wijk U, Carlsson I. Forearm amputees' views of prosthesis use and sensory feedback. J Hand Ther. 2015. doi: 10.1016/j.jht.2015.01.013.

13 Imaizumi S, Asai T, Koyama S. Embodied prosthetic arm stabilizes body posture, while unembodied one perturbs it. Conscious Cogn. 2016. doi: 10.1016/j.concog.2016.08.019.

14 Lebedev MA, Nicolelis MA. Brain-Machine Interfaces: From Basic Science to Neuroprostheses and Neurorehabilitation. Physiol Rev. 2017. doi: 10.1152/physrev.00027.2016.

15 Sebelius FC, Rosén BN, Lundborg GN. Refined myoelectric control in below-elbow amputees using artificial neural networks and a data glove. J Hand Surg Am. 2005. doi: 10.1016/j.jhsa.2005.01.002.

16 Maruishi M, Tanaka Y, Muranaka H, Tsuji T, Ozawa Y, Imaizumi S, Miyatani M, Kawahara J. Brain activation during manipulation of the myoelectric prosthetic hand: a functional magnetic resonance imaging study. Neuroimage. 2004. doi: 10.1016/j.neuroimage.2003.12.001.

17 Kluger DT, Joyner JS, Wendelken SM, Davis TS, George JA, Page DM, Hutchinson DT, Benz HL, Clark GA. Virtual Reality Provides an Effective Platform for Functional Evaluations of Closed-Loop Neuromyoelectric Control. IEEE Trans Neural Syst Rehabil Eng. 2019. doi: 10.1109/TNSRE.2019.2908817. 18 Qian J, McDonough DJ, Gao Z. The Effectiveness of Virtual Reality Exercise on Individual's Physiological, 
Psychological and Rehabilitative Outcomes: A Systematic Review. Int J Environ Res Public Health. 2020. doi: 10.3390/ijerph17114133.

19 Ehrsson HH, Rosén B, Stockselius A, Ragnö C, Köhler P, Lundborg G. Upper limb amputees can be induced to experience a rubber hand as their own. Brain. 2008. doi: 10.1093/brain/awn297.

20 Antfolk C, D'Alonzo M, Controzzi M, Lundborg G, Rosén B, Sebelius F, Cipriani C. Artificial redirection of sensation from prosthetic fingers to the phantom hand map on transradial amputees: vibrotactile versus mechanotactile sensory feedback. IEEE Trans Neural Syst Rehabil Eng. 2013. doi: 10.1109/TNSRE.2012.2217989.

21 D'Alonzo M, Clemente F, Cipriani C. Vibrotactile stimulation promotes embodiment of an alien hand in amputees with phantom sensations. IEEE Trans Neural Syst Rehabil Eng. 2015. doi: 10.1109/TNSRE.2014.2337952.

22 Jackson, AEEF. Interfacing with the Computational Brain. Bone. 2008. https://doi.org/10.1109/TNSRE.2011.2158586.

23 Sime DW. Potential Application of Virtual Reality for Interface Customisation (and Pre-training) of Amputee Patients as Preparation for Prosthetic Use. Adv Exp Med Biol. 2019. doi: 10.1007/978-3-030-06070$1 \_2$.

24 Bohil CJ, Alicea B, Biocca FA. Virtual reality in neuroscience research and therapy. Nat Rev Neurosci. 2011. doi: $10.1038 / \mathrm{nrn} 3122$.

25 Gumaa M, Rehan Youssef A. Is Virtual Reality Effective in Orthopedic Rehabilitation? A Systematic Review and Meta-Analysis. Phys Ther. 2019. doi: 10.1093/ptj/pzz093.

26 Slater M, Perez-Marcos D, Ehrsson HH, Sanchez-Vives MV. Inducing illusory ownership of a virtual body. Front Neurosci. 2009. doi: 10.3389/neuro.01.029.2009.

27 Slater M, Spanlang B, Sanchez-Vives MV, Blanke O. First person experience of body transfer in virtual reality. PLoS One. 2010. doi: 10.1371/journal.pone.0010564.

28 Sengül A, van Elk M, Rognini G, Aspell JE, Bleuler H, Blanke O. Extending the body to virtual tools using a robotic surgical interface: evidence from the crossmodal congruency task. PLoS One. 2012. doi: 10.1371/journal.pone.0049473.

29 Shokur S, Gallo S, Moioli RC, Donati ARC, Morya E, Bleuler H, Nicolelis MAL. Assimilation of virtual legs and perception of floor texture by complete paraplegic patients receiving artificial tactile feedback. Sci Rep. 2016. doi: 10.1038/srep32293.

30 Proske U, Gandevia SC. The proprioceptive senses: their roles in signaling body shape, body position and movement, and muscle force. Physiol Rev. 2012. doi: 10.1152/physrev.00048.2011.

31 Butler AA, Héroux ME, Gandevia SC. Body ownership and a new proprioceptive role for muscle spindles. Acta Physiol (Oxf). 2017. doi: 10.1111/apha.12792.

32 Riemann BL, Lephart SM. The Sensorimotor System, Part II: The Role of Proprioception in Motor Control and Functional Joint Stability. J Athl Train. 2002 Jan;37(1):80-4.

33 Pedrinelli A. Tratamento do paciente com amputação. 1 ed. São Paulo: Roca, 2004.

34 Jensen, Mark P., Paul Karoly, and Sanford Braver. The measurement of clinical pain intensity: a comparison of six methods. Pain. 1986: 117-126.

35 Hawker GA, Mian S, Kendzerska T, French M. Measures of adult pain: Visual Analog Scale for Pain (VAS Pain), Numeric Rating Scale for Pain (NRS Pain), McGill Pain Questionnaire (MPQ), Short-Form McGill Pain Questionnaire (SF-MPQ), Chronic Pain Grade Scale (CPGS), Short Form-36 Bodily Pain Scale (SF-36 BPS), and Measure of Intermittent and Constant Osteoarthritis Pain (ICOAP). Arthritis Care Res (Hoboken). 2011. doi: 10.1002/acr.20543.

36 Mentiplay BF, Perraton LG, Bower KJ, Adair B, Pua YH, Williams GP, McGaw R, Clark RA. Assessment of Lower Limb Muscle Strength and Power Using Hand-Held and Fixed Dynamometry: A Reliability and Validity Study. PLoS One. 2015. doi: 10.1371/journal.pone.0140822.

37 Gailey RS, Roach KE, Applegate EB, Cho B, Cunniffe B, Licht S, Maguire M, Nash MS. The amputee 
mobility predictor: an instrument to assess determinants of the lower-limb amputee's ability to ambulate. Arch Phys Med Rehabil. 2002. doi: 10.1053/ampr.2002.32309.

38 Matsudo S, Araujo T, Matsudo V, Andrade D, Andrade E, et al. Questionário Internacional de Atividade Fisica (IPAQ): Estudo de validade e reprodutibilidade no Brasil. Atividade Física e Saúde 2001; 6 (2): 5-18.

39 Sarmento A. Apresentação e aplicabilidade da versão brasileira da MoCA (Montreal Cognitive Assessment) para rastreio de Comprometimento Cognitivo Leve Tese apresentada à Universidade Federal de São PauloEscola Paulista de Medicina, para obtenção do Título de Mestre. 2009.

40 Botega NJ, Bio MR, Zomignani MA, Garcia C Jr, Pereira WA. Transtornos do humor em enfermaria de clínica médica e validação de escala de medida (HAD) de ansiedade e depressão [Mood disorders among inpatients in ambulatory and validation of the anxiety and depression scale HAD]. Rev Saude Publica. 1995. doi: 10.1590/s0034-89101995000500004.

41 Kendall, F. P., McCreary, E. K., Provance, P. G. Músculos provas e funções. 4a edição. ed. São Paulo: Manole, 1995.

42 Black C, Voigts J, Agrawal U, Ladow M, Santoyo J, Moore C, Jones S. Open Ephys electroencephalography (Open Ephys + EEG): a modular, low-cost, open-source solution to human neural recording. J Neural Eng. 2017. doi: 10.1088/1741-2552/aa651f.

43 Siegle JH, López AC, Patel YA, Abramov K, Ohayon S, Voigts J. Open Ephys: an open-source, pluginbased platform for multichannel electrophysiology. J Neural Eng. 2017. doi: 10.1088/1741-2552/aa5eea.

44 Moreira, J.V.S. et al. Electromyography biofeedback system with visual and vibratory feedbacks designed for lower limb rehabilitation. 2021. arXiv:2103.04034.

45 Stegeman, D., \& Hermens, H. Standards for surface electromyography: The European project Surface EMG for non-invasive assessment of muscles (SENIAM). 2007.

46 Staudenmann D, Roeleveld K, Stegeman DF, van Dieën JH. Methodological aspects of SEMG recordings for force estimation--a tutorial and review. J Electromyogr Kinesiol. 2010. doi: 10.1016/j.jelekin.2009.08.005.

47 Jones LA, Kunkel J, Piateski E. Vibrotactile pattern recognition on the arm and back. Perception. 2009. doi: 10.1068/p5914.

48 Kandel, E. R., Schwartz, J. H., Jessell, T. M., Siegelbaum, S. A., \& Hudspeth, A. J.. Princípios de Neurociências. 5 ${ }^{\text {a }}$ Ed. 1-1531. 2014.

49 SENIAM - Surface ElectroMyoGraphy for the Non-Invasive Assessment of Muscles. Available at: http://www.seniam.org. Access on: January 2020.

50 Teplan, M. Fundamentals of EEG measurement. Measurement Science Review. 2(2), 1-11 2002.

51 Stern, J. M. Atlas of EEG Patterns. Lippincott Williams \& Wilkins, a Wolters Kluwer business, Philadelphia. 2015.

52 Skinner, B. F. The Behavior of Organisms: An Experimental Analysis. New York: Appleton-Century-Crofts. 1938.

53 Critchley HD. Electrodermal responses: what happens in the brain. Neuroscientist. 2002. doi: $10.1177 / 107385840200800209$.

54 Armel KC, Ramachandran VS. Projecting sensations to external objects: evidence from skin conductance response. Proc Biol Sci. 2003. doi: 10.1098/rspb.2003.2364.

55 Alimardani M, Nishio S, Ishiguro H. Removal of proprioception by BCI raises a stronger body ownership illusion in control of a humanlike robot. Sci Rep. 2016. doi: 10.1038/srep33514.

56 Marini F, Tagliabue CF, Sposito AV, Hernandez-Arieta A, Brugger P, Estévez N, Maravita A. Crossmodal representation of a functional robotic hand arises after extensive training in healthy participants. Neuropsychologia. 2014. doi: 10.1016/j.neuropsychologia.2013.11.017.

57 Shumway-Cook, A., Woollacott, M. H.. Controle Motor Teoria e aplicações práticas. 2010.

58 Buzsáki, G. Rhythms of the Brain. In Rhythms of the Brain. 2009.

59 Braithwaite, J. J., Watson, D. G., Jones, R., \& Rowe, M. A Guide for Analysing Electrodermal Activity \& Skin Conductance Responses (SCRs) for Psychophysiological Experiments. Via the Biopac MP36R \& 
AcqKnowledge software. 2015.

60 Yuan, Ye, and Anthony Steed. Is the rubber hand illusion induced by immersive virtual reality?. 2010 IEEE Virtual Reality Conference (VR).

61 Maravita A, Spence C, Driver J. Multisensory integration and the body schema: close to hand and within reach. Curr Biol. 2003. doi: 10.1016/s0960-9822(03)00449-4.

62 Tsakiris M, Hesse MD, Boy C, Haggard P, Fink GR. Neural signatures of body ownership: a sensory network for bodily self-consciousness. Cereb Cortex. 2007. doi: 10.1093/cercor/bhl131.

63 Tsakiris M, Schütz-Bosbach S, Gallagher S. On agency and body-ownership: phenomenological and neurocognitive reflections. Conscious Cogn. 2007. doi: 10.1016/j.concog.2007.05.012.

64 Delorme, A. et al.. EEGLAB, SIFT, NFT, BCILAB, and ERICA: New tools for advanced EEG processing. Computational Intelligence and Neuroscience. 2011.

65 Petre, S., Moses, R. Spectral Analysis of Signals. Upper Saddle River, NJ: Prentice Hall. 2005.

66 Marsaglia, George, Wai Wan Tsang, and Jingbo Wang. "Evaluating Kolmogorov's distribution." Journal of statistical software. 2003: 1-4.

67 McLachlan, G.J.. Discriminant Analysis and Statistical Pattern Recognition, John Wiley \& Sons, New York, N.Y.1992.

68 Dobson, A. J., Barnett, A. G. An Introduction to Generalized Linear Models. Chapman and Hall/CRC. Taylor \& Francis Group. 2008.

69 Tieri G, Tidoni E, Pavone EF, Aglioti SM. Body visual discontinuity affects feeling of ownership and skin conductance responses. Sci Rep. 2015. doi: 10.1038/srep17139.

70 Fusaro M, Tieri G, Aglioti SM. Seeing pain and pleasure on self and others: behavioral and psychophysiological reactivity in immersive virtual reality. J Neurophysiol. 2016. doi: 10.1152/jn.00489.2016. 71 Serino A, Bassolino M, Farnè A, Làdavas E. Extended multisensory space in blind cane users. Psychol Sci. 2007. doi: 10.1111/j.1467-9280.2007.01952.x.

72 Bassolino M, Serino A, Ubaldi S, Làdavas E. Everyday use of the computer mouse extends peripersonal space representation. Neuropsychologia. 2010. doi: 10.1016/j.neuropsychologia.2009.11.009.

73 Koessler L, Maillard L, Benhadid A, Vignal JP, Felblinger J, Vespignani H, Braun M. Automated cortical projection of EEG sensors: anatomical correlation via the international 10-10 system. Neuroimage. 2009. doi: 10.1016/j.neuroimage.2009.02.006.

74 Jiang J, Zhang Q, van Gaal S. Conflict awareness dissociates theta-band neural dynamics of the medial frontal and lateral frontal cortex during trial-by-trial cognitive control. Neuroimage. 2015. doi: 10.1016/j.neuroimage.2015.04.062.

75 Sauseng P, Griesmayr B, Freunberger R, Klimesch W. Control mechanisms in working memory: a possible function of EEG theta oscillations. Neurosci Biobehav Rev. 2010. doi: 10.1016/j.neubiorev.2009.12.006.

76 Inanaga K. Frontal midline theta rhythm and mental activity. Psychiatry Clin Neurosci. 1998. doi: 10.1046/j.1440-1819.1998.00452.x.

77 Ishii R, Canuet L, Ishihara T, Aoki Y, Ikeda S, Hata M, Katsimichas T, Gunji A, Takahashi H, Nakahachi T, Iwase M, Takeda M. Frontal midline theta rhythm and gamma power changes during focused attention on mental calculation: an MEG beamformer analysis. Front Hum Neurosci. 2014. doi: 10.3389/fnhum.2014.00406. 78 Pfurtscheller G, Stancák A Jr, Edlinger G. On the existence of different types of central beta rhythms below 30 Hz. Electroencephalogr Clin Neurophysiol. 1997. doi: 10.1016/s0013-4694(96)96612-2.

79 Holmes NP, Spence C. The body schema and the multisensory representation(s) of peripersonal space. Cogn Process. 2004. doi: 10.1007/s10339-004-0013-3.

80 Dijkerman HC, de Haan EH. Somatosensory processes subserving perception and action. Behav Brain Sci. 2007. doi: 10.1017/S0140525X07001392.

81 Kanayama N, Sato A, Ohira H. Crossmodal effect with rubber hand illusion and gamma-band activity. Psychophysiology. 2007. doi: 10.1111/j.1469-8986.2007.00511.x.

82 Kanayama N, Sato A, Ohira $\mathrm{H}$. The role of gamma band oscillations and synchrony on rubber hand illusion 
and crossmodal integration. Brain Cogn. 2009. doi: 10.1016/j.bandc.2008.05.001.

83 Anderson KL, Ding M. Attentional modulation of the somatosensory mu rhythm. Neuroscience. 2011. doi: 10.1016/j.neuroscience.2011.02.004.

84 Freyer F, Reinacher M, Nolte G, Dinse HR, Ritter P. Repetitive tactile stimulation changes resting-state functional connectivity-implications for treatment of sensorimotor decline. Front Hum Neurosci. 2012. doi: 10.3389/fnhum.2012.00144.

85 La'davas E. Functional and dynamic properties of visual peripersonal space. Trends Cogn Sci. 2002. doi: 10.1016/s1364-6613(00)01814-3.

86 Ehrsson HH, Spence C, Passingham RE. That's my hand! Activity in premotor cortex reflects feeling of ownership of a limb. Science. 2004. doi: 10.1126/science.1097011.

87 Ehrsson HH, Holmes NP, Passingham RE. Touching a rubber hand: feeling of body ownership is associated with activity in multisensory brain areas. J Neurosci. 2005. doi: 10.1523/JNEUROSCI.0800-05.2005.

88 Tsakiris M. My body in the brain: a neurocognitive model of body-ownership. Neuropsychologia. 2010. doi: 10.1016/j.neuropsychologia.2009.09.034.

89 Brozzoli C, Gentile G, Ehrsson HH. That's near my hand! Parietal and premotor coding of hand-centered space contributes to localization and self-attribution of the hand. J Neurosci. 2012. doi: 10.1523/JNEUROSCI.2660-12.2012.

90 Haggard P. Sense of agency in the human brain. Nat Rev Neurosci. 2017. doi: 10.1038/nrn.2017.14.

91 Wolpert DM, Diedrichsen J, Flanagan JR. Principles of sensorimotor learning. Nat Rev Neurosci. 2011. doi: $10.1038 / \mathrm{nrn} 3112$.

92 Wolpert DM, Ghahramani Z, Jordan MI. An internal model for sensorimotor integration. Science. 1995. doi: 10.1126/science. 7569931 .

93 Wolpert DM, Ghahramani Z. Computational principles of movement neuroscience. Nat Neurosci. 2000. doi: $10.1038 / 81497$.

94 Giggins OM, Persson UM, Caulfield B. Biofeedback in rehabilitation. J Neuroeng Rehabil. 2013. doi: 10.1186/1743-0003-10-60. 


\section{Additional files}

Additional file 1 - Participants' reports regarding their main complaints, expectations, and general comments.

\begin{tabular}{|c|c|c|c|}
\hline \multirow{2}{*}{ Participants } & \multicolumn{3}{|c|}{ Reports } \\
\hline & Main complaint & Expectancy & Comments \\
\hline A & "I can't drive" & $\begin{array}{l}\text { "I can use the prosthesis and } \\
\text { adapt" }\end{array}$ & $\begin{array}{c}\text { "I had a phantom limb } \\
\text { sensation, it stopped two } \\
\text { months ago" }\end{array}$ \\
\hline B & "I'm very tired" & "I get more independent" & $\begin{array}{l}\text { "For the first time in } 8 \text { years I get } \\
\text { to bend my knee"*1 }\end{array}$ \\
\hline C & "I can't ride a motorcycle" & "Prosthetic fitting" & $\begin{array}{l}\text { Initial session - "I rarely feel the } \\
\text { phantom limb. Final session -" } \\
\text { The phantom sensation is rare, } \\
\text { sometimes I can control the } \\
\text { phantom limb" }\end{array}$ \\
\hline D & $\begin{array}{c}\text { "Use the crutch, both arms are } \\
\text { occupied" }\end{array}$ & "Prosthetic fitting" & Did not make other reports \\
\hline $\mathrm{E}$ & $\begin{array}{c}\text { "Difficulty taking a license from } \\
\text { work" }\end{array}$ & $\begin{array}{c}\text { "Win a prosthesis and use it for } \\
\text { everything" }\end{array}$ & $\begin{array}{c}\text { "I feel and can control my } \\
\text { phantom limb". "When I bend } \\
\text { the virtual prosthesis, I do it the } \\
\text { same way when I want to bend } \\
\text { the phantom knee"*2 }\end{array}$ \\
\hline $\mathrm{F}$ & "Depend on other people" & $\begin{array}{c}\text { "Get the prosthesis and do } \\
\text { everything" }\end{array}$ & $\begin{array}{c}\text { "It's like I have two legs } \\
\text { moving"*3 }\end{array}$ \\
\hline G & "Back to work" & "Get the prosthesis" & $\begin{array}{c}\text { "I felt the phantom limb only in } \\
\text { the first three months after the } \\
\text { amputation" }\end{array}$ \\
\hline
\end{tabular}

*1 This participant had a history of traumatic injury to the lower limb with a series of complications during the attempt to save the limb, culminating in knee arthrodesis and finally in the amputation decision. This whole process up to the surgical amputation procedure lasted approximately 8 years. *2 During the first days of training, he reported greater difficulty performing knee flexion of the virtual prosthesis.

*3 She reported a sensation of a phantom limb, as if the knee was constantly flexed. She tried to control it, but she could not. During training with the EMG-based HMI and immersion in the VR environment, 
she reported that when she exerted strength to flex the virtual prosthesis knee, she felt that along with the prosthesis, the phantom limb also flexed.

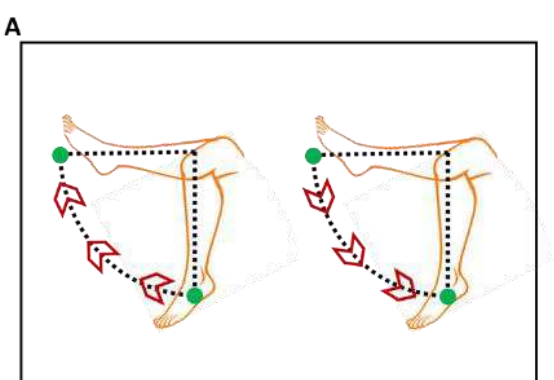

A.1

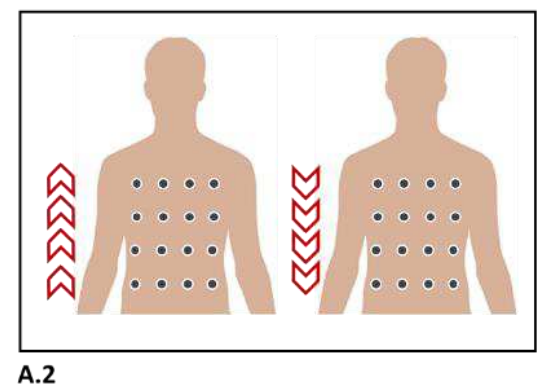

B

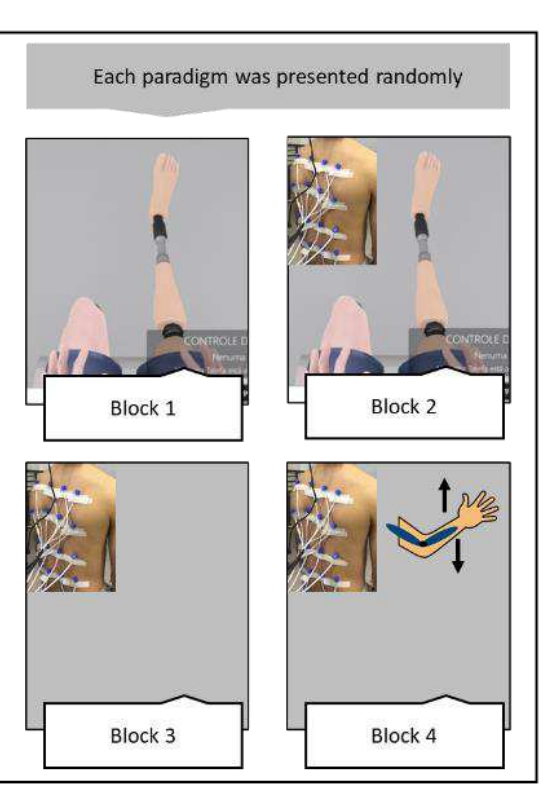

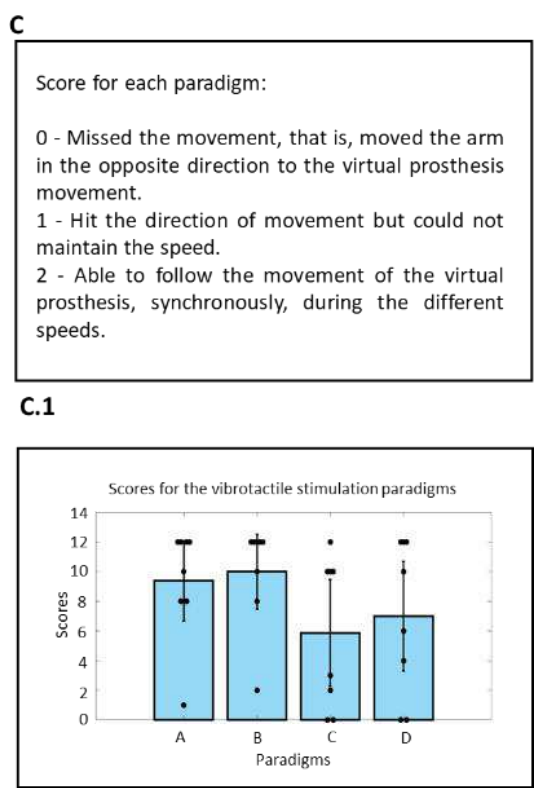

C.2

Additional file 2 - Identification of the vibrotactile stimulation pattern. A) Possible paradigms: four different combinations (knee extension and upward vibratory stimulation; knee extension and downward vibratory stimulation; knee flexion and downward vibratory stimulation; knee flexion and upward vibratory stimulation) A.1) Movements of the virtual prosthesis: knee extension and flexion. A.2) Application of upward and downward vibrotactile stimuli. B) The presentation sequence of the paradigms was random, and for each one, the following protocol was followed: Block 1 - participants observed the movements of the virtual prosthesis at a speed of $45 \%$, without vibrotactile feedback, for 30 s. Block 2 - participants received vibratory stimuli on the back in sync with the avatar movements based on a predefined combination for $60 \mathrm{~s}$. Block 3 - VR glasses were turned off, but the virtual prosthesis continued to move, and the vibrotactile stimulation pattern corresponding to the movement was provided. The participants were instructed to trust vibrotactile stimulation while imagining the virtual prosthesis moving (30 s). Block 4 - VR glasses remained off, but the virtual prosthesis continued to move at different speeds (low $=30 \%$ s; average $=45 \%$ s and high $=180 \%$ s), with corresponding vibrotactile feedback. Participants were instructed to move their arm homolaterally to the amputation at the same speed to reproduce the movements that the prosthesis performed, guided only by vibratory stimuli. For this, a goniometer was attached to the elbow joint to detect movement. In this manner, when the participants wished to represent the extension movement of the virtual prosthesis (leg elevation), they should perform elbow flexion (arm elevation), and the opposite was true for the reverse movement. C) Final score. C.1) Criteria adopted for scoring in each paradigm. C.2) Graph with the final scores (median and CIs) for all participants in each paradigm (A- downward vibration to represent flexion; B- upward vibration to represent extension; C- upward vibration to represent flexion; Ddownward vibration to represent extension). The vibrotactile stimulation pattern that generated the highest score in the recognition of each movement performed by the virtual prosthesis was chosen for 
the training protocol (in the case of a tie, the vibrotactile stimulation pattern that followed the same direction of virtual prosthesis movement was selected).

A

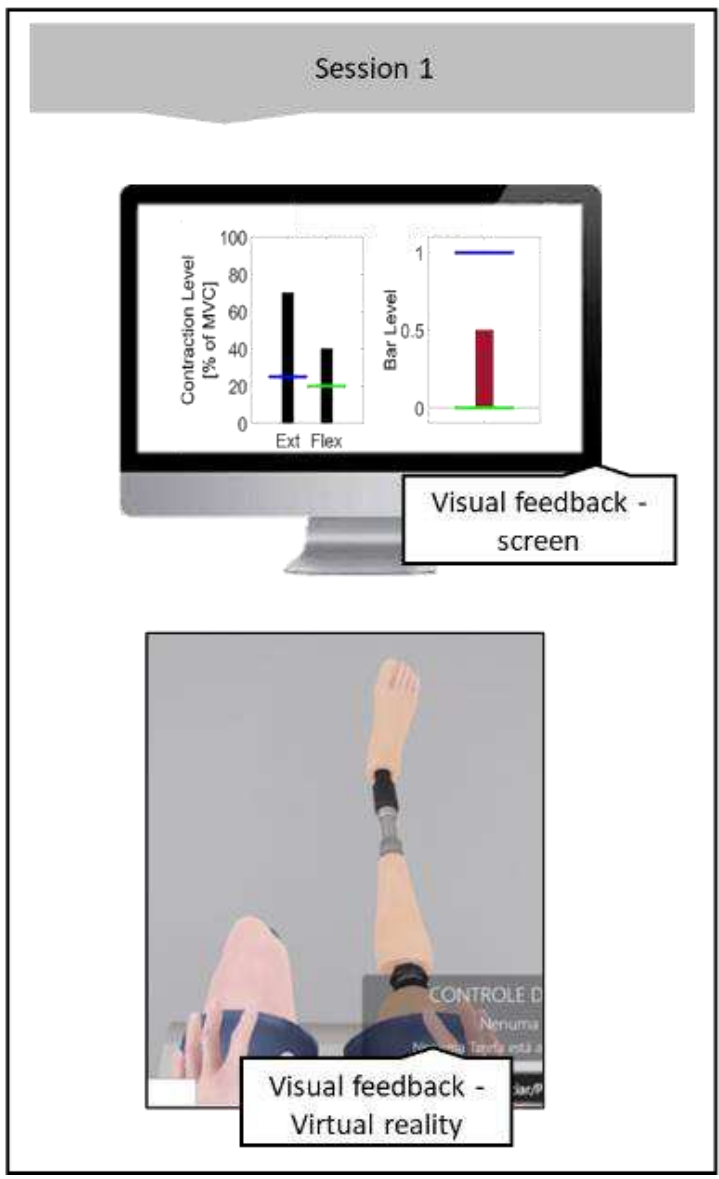

B

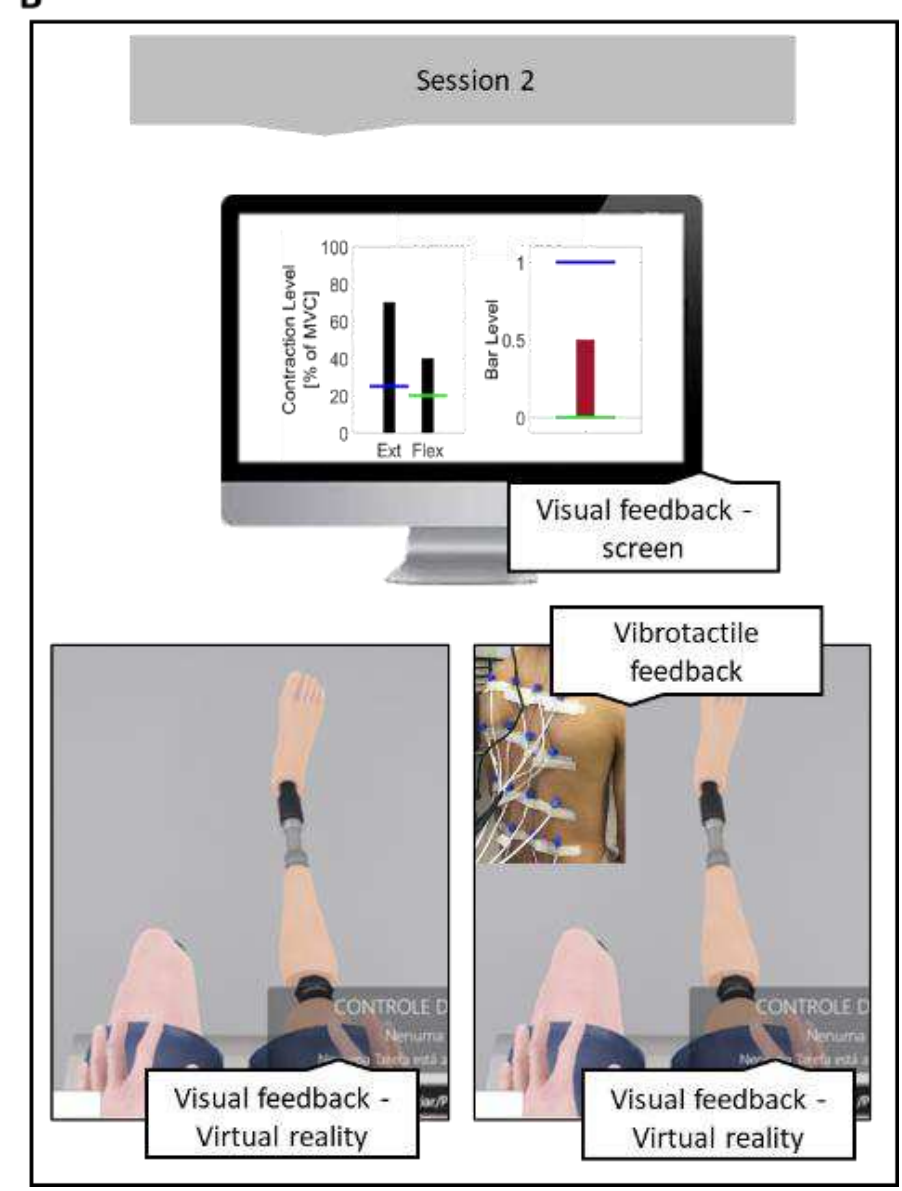

Additional file 3 - Familiarization sessions. A) Session 1: Visual feedback on the screen (bars) - four repetitions were performed (in each of them, the participant had to perform four movements up and down the bar). Then, a repetition was performed with visual feedback in the virtual environment, consisting of ten free knee extension and flexion movements of the virtual prosthesis. B) Session 2: Visual feedback on the screen (bars) - three repetitions (five movements up and down the bar). Visual feedback in the virtual environment - three repetitions of free knee extension and flexion movements of the virtual prosthesis. Afterward, the same protocol was repeated with the association of vibratory stimuli on the back to represent the virtual prosthesis movements. In these sessions, the control parameters consisted of muscle activation above $2 \mathrm{SD}$ of the mean baseline signal and antagonist muscle contraction tolerance of $80 \%$. 
A

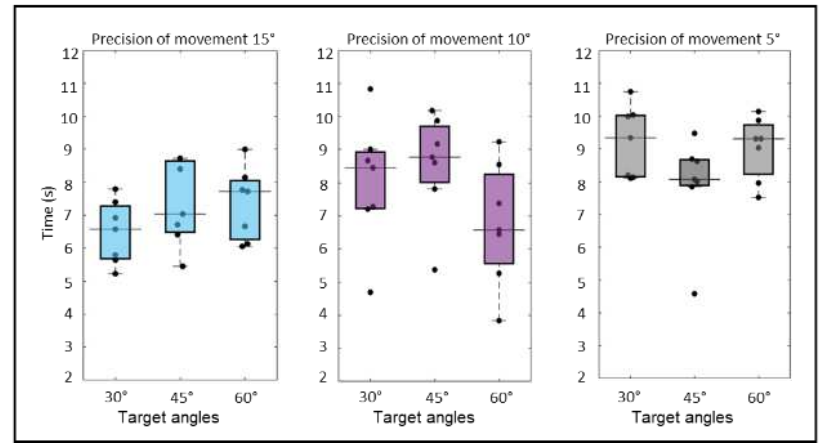

B

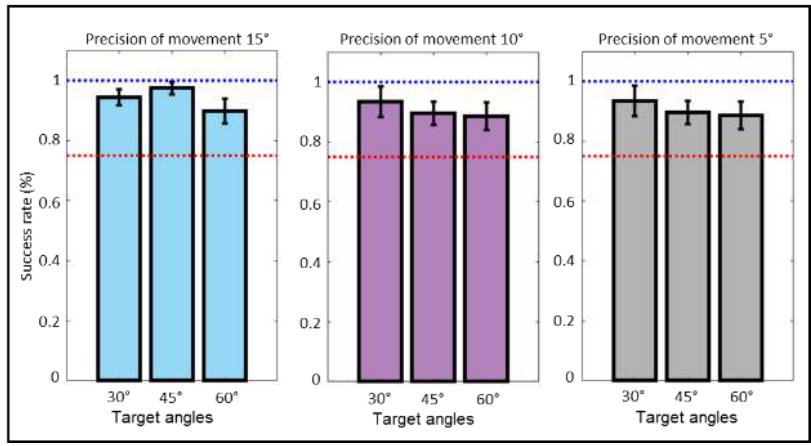

Additional file 4 - Analysis of intermediate target angles of $30^{\circ}, \mathbf{4 5}^{\circ}$ and $60^{\circ}$. A) Execution time for each difficulty level of movement precision (Kruskal-Wallis with Tukey-Kramer correction). B) Success rate for each difficulty level related to movement precision (average proportions and CIs). The red line indicates a success rate of $75 \%$, and the blue line indicates a success rate of $100 \%$.

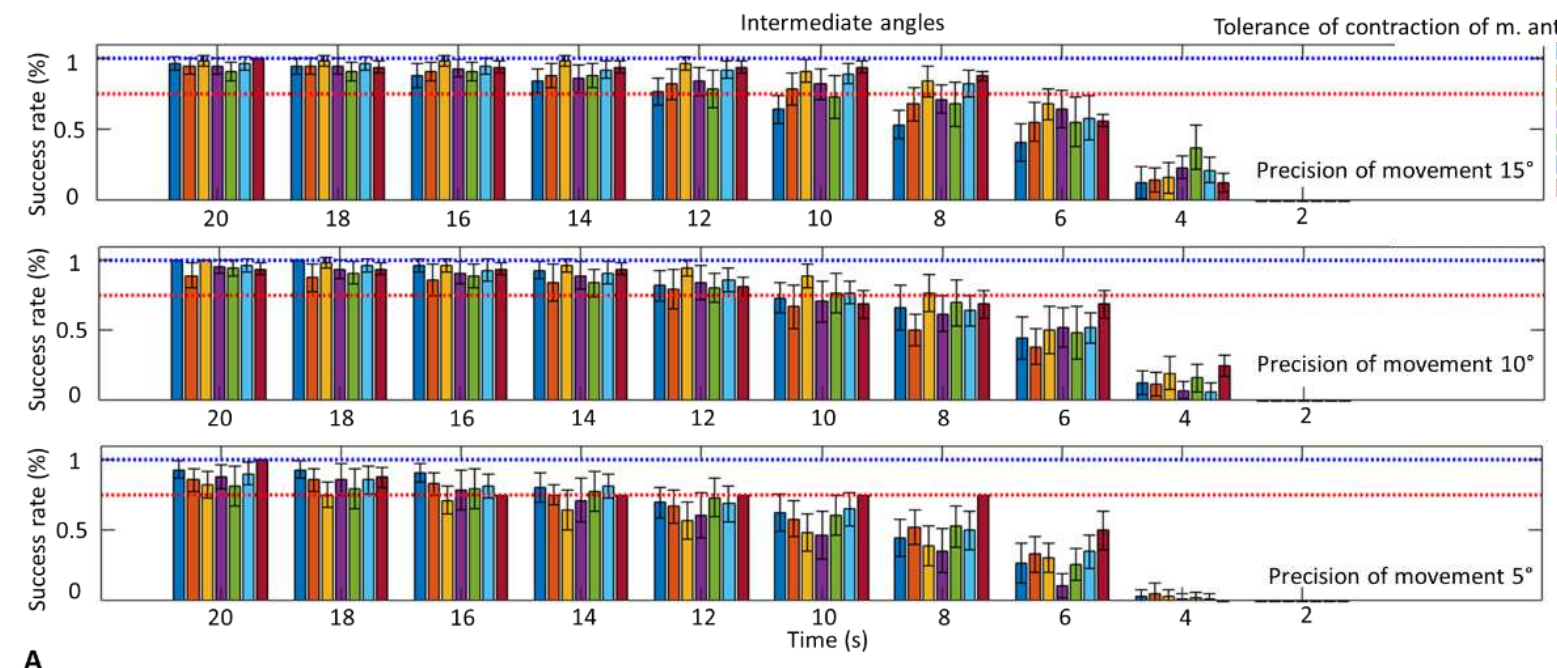

A
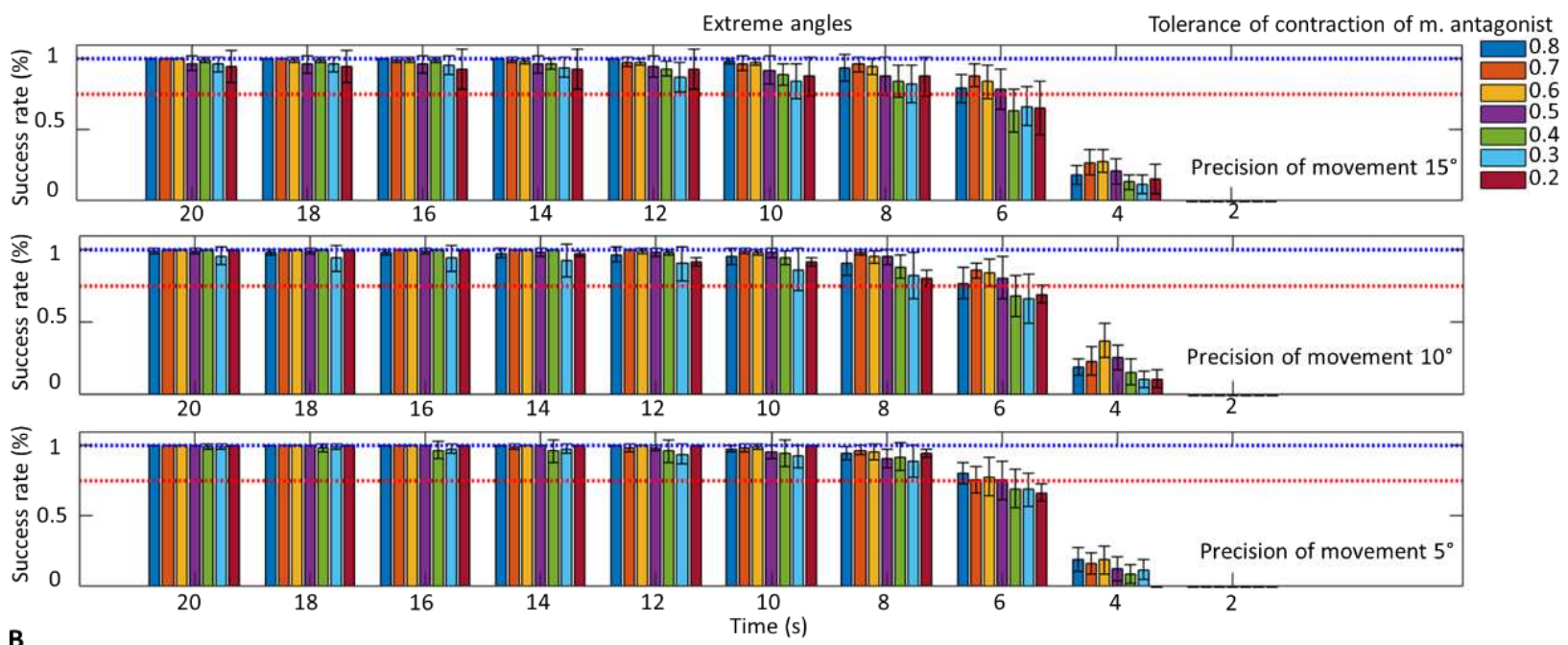

B

Additional file 5 - Success rate during training with the EMG-based HMI (visual and vibrotactile stimulation) across different time thresholds. A) Success rate (average proportions and CIs) intermediate target angles $\left(30^{\circ}\right.$ and $\left.60^{\circ}\right)$. B) Success rate (average proportions and CIs) - extreme target 
angles $\left(0^{\circ}\right.$ and $\left.90^{\circ}\right)$. The blue line indicates a success rate of $100 \%$, and the red line indicates a success rate of $75 \%$.

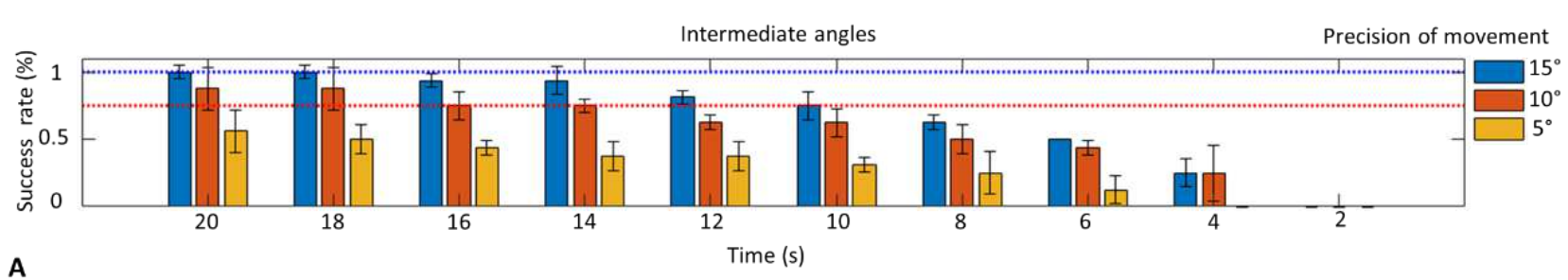

A

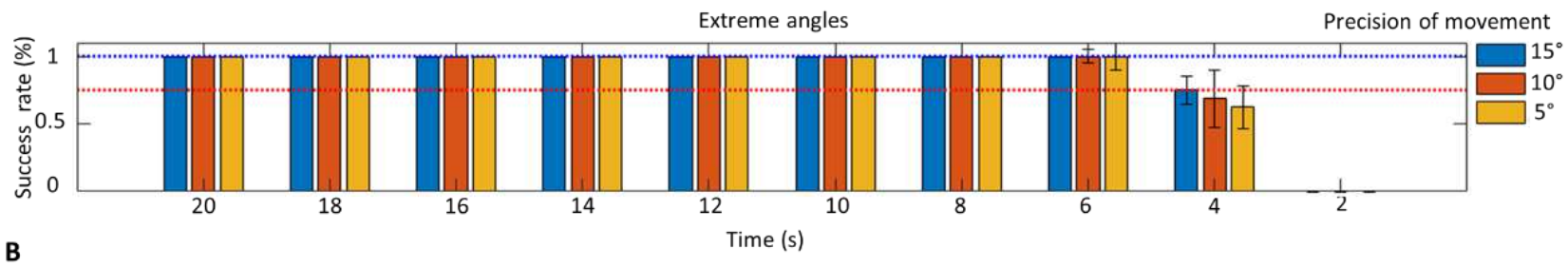

Additional file 6 - Success rate during the perception evaluation test of virtual prosthesis movements using vibrotactile stimuli without the aid of vision across the different time thresholds. A) Success rate (average proportions and CIs) - intermediate target angles $\left(30^{\circ}\right.$ and $\left.60^{\circ}\right)$. B) Success rate (average proportions and CIs) - extreme target angles $\left(0^{\circ}\right.$ and $\left.90^{\circ}\right)$. The blue line indicates a success rate of $100 \%$, and the red line indicates a success rate of $75 \%$. 


\section{Supplementary Files}

This is a list of supplementary files associated with this preprint. Click to download.

- CAREchecklist.pdf 